\title{
24. PHYSICAL PROPERTIES AND ELASTIC CONSTANTS OF UPPER CRUSTAL ROCKS FROM CORE-LOG MEASUREMENTS IN HOLE 504B ${ }^{1}$
}

\author{
Gerardo J. Iturrino, ${ }^{2}$ Nikolas I. Christensen, ${ }^{3}$ Keir Becker, ${ }^{2}$ \\ Lars O. Boldreel, ${ }^{4}$ Peter K.H. Harvey, ${ }^{5}$ and Philippe Pezard ${ }^{6}$
}

\begin{abstract}
Seismic velocities have been measured at confining pressures of $100 \mathrm{MPa}$ and $600 \mathrm{MPa}$ for sheeted dike samples recovered during Ocean Drilling Program Legs 137 and 140. The compressional- and shear-wave velocities show an increase with depth at Hole 504B, which is in sharp contrast to the atmospheric pressure velocity measurements performed as part of the shipboard analyses. Rocks exposed to different types of alteration and fracture patterns show distinct changes in their physical properties. The seismic reflectors observed on the vertical seismic profile (VSP) experiment performed during Leg 111 may have been caused by low velocity zones resulting from alteration. The amount of fracturing and hydrothermal alteration in several zones also may have contributed to the acoustic impedance contrast necessary to produce the $\mathrm{E}_{5}$ reflector. Poisson's ratios calculated from laboratory velocity measurements show several low values at depths ranging from $1600 \mathrm{mbsf}$ to $2000 \mathrm{mbsf}$, which tends to follow similar trends obtained from previous oceanic refraction experiments. A comparison of physical properties between samples recovered from Hole 504B and ophiolite studies in the Bay of Islands and Oman shows a good correlation with the Bay of Islands but significant differences from the measurements performed in the Oman complex.
\end{abstract}

\section{INTRODUCTION}

Ocean Drilling Program (ODP) Leg 140 revisited Hole 504B, located at $1^{\circ} 13.611^{\prime} \mathrm{N}, 83^{\circ} 43.818^{\prime} \mathrm{W}$ or approximately $200 \mathrm{~km}$ south of the Costa Rica Rift in the eastern equatorial Pacific (Fig. 1). Leg 140 deepened the hole by $378.5 \mathrm{~m}$ to an approximate total depth of $2000 \mathrm{mbsf}$, more than three times as deep into the oceanic crust as the penetration of any other hole. The 5.9-m.y.-old crust at this site represents a section of oceanic crust formed at the Costa Rica Rift and extending through a sedimentary cover $(274.5 \mathrm{~m})$, and a section of extrusive pillow lavas with minor flows $(571.5 \mathrm{~m})$, which is underlain by a $209-\mathrm{m}$ zone of transition into the underlying sheeted dikes and massive units ( $945 \mathrm{~m})$. Therefore, this site is the best reference for the evolution, structure, and composition of the upper oceanic crust and represents an excellent opportunity to measure the physical properties and test the geophysical models of a relatively young crustal section.

Site survey seismic and heat-flow measurements (Langseth, et al., 1983; Hobart et al., 1985; Collins et al., 1989) as well as downhole temperature, porosity, and permeability data (Anderson et al., 1985; Becker, 1985 and 1989) show some particularly interesting aspects of the evolution of the ocean crust at Hole 504B. First, this relatively young crustal section has a thick sedimentary cover that, for the most part, seals the basement from pervasive hydrothermal circulation; crustal temperatures vary within a range of values that are consistent with predicted conductive heat transfer values (Parsons and Sclater, 1977; Langseth et al., 1983; Hobart et al., 1985; Langseth et al., 1988). Detailed heat-flow work and numerical simulations indicate that convection occurs in the permeable, uppermost 200-300 m of basement beneath the impermeable sediment cover (Langseth et al.,

'Erzinger, J., Becker, K., Dick, H.J.B., and Stokking, L.B. (Eds.), 1995. Proc. ODP, Sci. Results, 137/140: College Station, TX (Ocean Drilling Program).

${ }^{2}$ Rosenstiel School of Marine and Atmospheric Sciences, Division of Marine Geology and Geophysics, University of Miami, 4600 Rickenbacker Causeway, Miami, FL 33149 1098, U.S.A.

${ }^{3}$ Department of Earth and Atmospheric Sciences, Purdue University, West Lafayette, IN 47907, U.S.A.

${ }^{4}$ Geological Survey of Denmark, Thoravej 8, DK-2400 Copenhagen, NV, Denmark

${ }^{5}$ Borehole Research, Department of Geology, University of Leicester, Leicester. LE1 7RH, United Kingdom.

${ }^{6}$ Institut Méditerranéan de Téchnologie, Téchnopole de Château-Gombert, 1345I Marseille Cedex 13, France.
1983; Fisher et al., 1990). This correlation is thought to be partially controlled by the presence of isolated basement faults and topographic highs.

Detailed studies of the velocity structures of ophiolites (Salisbury and Christensen, 1978) support seismic gradient models of the oceanic crust (Kempner and Gettrust, 1982) and provide a basis for interpretation of refraction velocities in terms of petrology. The rocks recovered during Legs 137 and 140 tend to support the ophiolite models and provide valuable samples for physical property studies of the oceanic crust. In this paper, we present densities and porosities, as well as elastic constants and seismic velocities as a function of confining pressure for the rock section drilled during Legs 137 and 140. The laboratory measurements of velocities and densities are compared with previous results from Hole 504B and ophiolite data from the Bay of Islands massifs as well as the Oman complex.

\section{EXPERIMENTAL METHODS}

The mineralogical composition, crack porosity, bulk density, degree of alteration, preferred mineral orientation, pore pressure, and temperature are among the important characteristics responsible for variations in seismic velocities throughout the oceanic crust. Because velocities at in-situ pressures are often different from velocities at atmospheric pressure (Birch, 1960), it is important that velocity data used for crustal studies be obtained at elevated pressures and temperatures.

The drill core recovered during Leg 140 was cut along its axis on board the ship while horizontal minicores were drilled normal to the drill core. Compressional- and shear-wave velocities $\left(V_{p}\right.$ and $V_{s}$, respectively) were measured on water-saturated samples at confining pressures of $100 \mathrm{MPa}$ and $600 \mathrm{MPa}$, using the pulse transmission method (Birch 1960; 1961); results are presented in Table 1. All of the $100 \mathrm{MPa}$ velocity measurements were performed at the Rosenstiel School of Marine and Atmospheric Science using a compound ultrasonic transducer system (Fig. 2) with velocity determinations accurate to approximately $1 \%$. This system is capable of simultaneously measuring a $V_{p}$ and two orthogonally polarized shear-wave velocities as a function of confining pressure. The minicores were prepared with both sides flat and parallel to within $0.01 \mathrm{~mm}$ and having the same dimensions as the shipboard samples (Dick, Erzinger, Stokking, et al., 1992). The samples were placed on $1-\mathrm{MHz}$ transducers, and rubber 


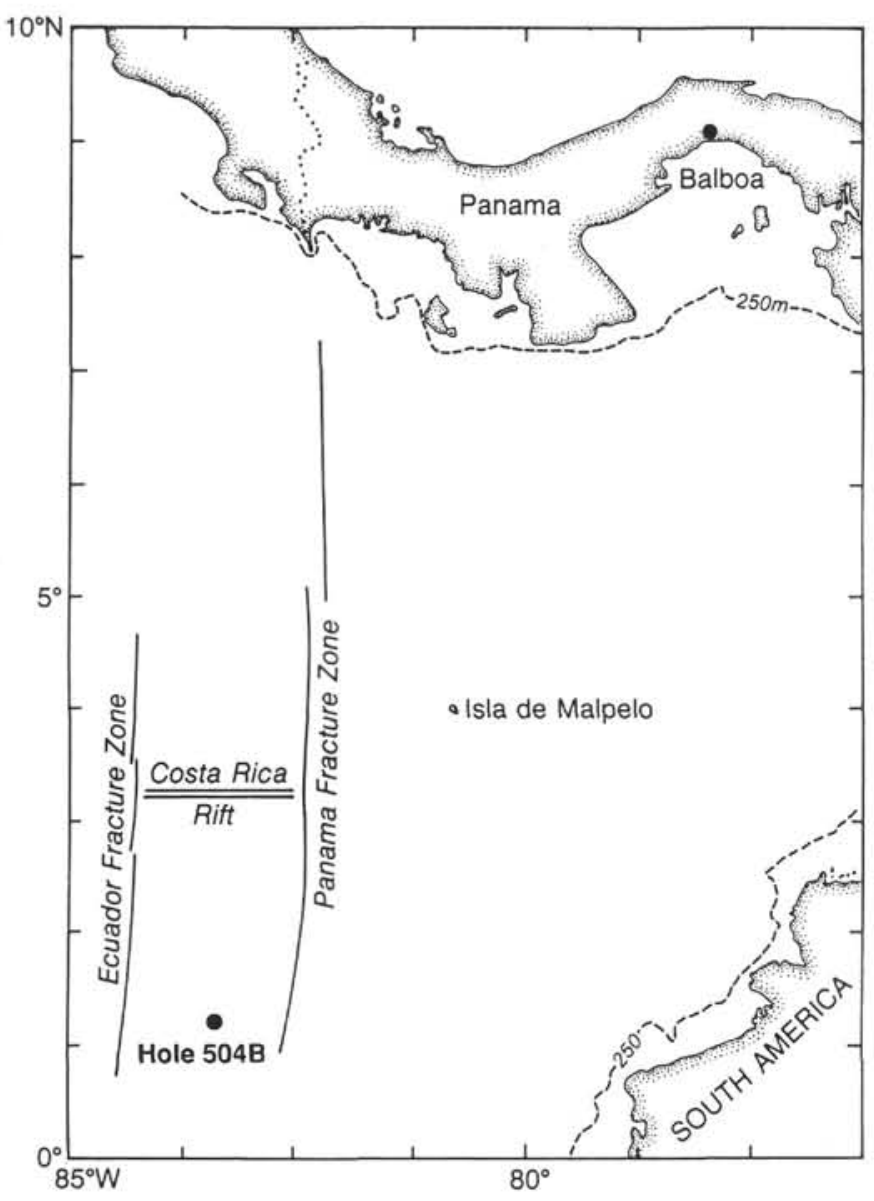

Figure 1. Location map of Hole 504B.

tubing was fitted and clamped over the assembly to prevent the pressure medium from invading the pore spaces. All measurements were obtained at room temperature and constant pore pressure of 2 $\mathrm{MPa}$ to keep the samples saturated during the measurements while avoiding pore pressure build-up.

$V_{p}$ and $V_{s}$ measurements as a function of confining pressure of 10 samples were performed at the Purdue University Rock Physics Laboratory at pressures up to $600 \mathrm{MPa}$ using the pulse transmission method (Christensen 1965, 1985; Iturrino et al., 1991). This method also determines the traveltime of an elastic wave in a rock cylinder of known length and yields velocities accurate to approximately $1 \%$.

Wet and dry sample weights and volumes were determined using a motion-compensated microbalance for measuring mass $( \pm 0.002 \mathrm{~g} \mathrm{ac}-$ curacy) and a pycnometer for measuring sample volumes $( \pm 0.03 \%)$. The pycnometer is specifically designed to measure the volume and to provide the necessary information to calculate the density, porosity, and water content of the samples by employing Archimedes' principle of fluid displacement. The displaced fluid is helium, which assures penetration into crevices and pore spaces approaching one Angstrom $\left(10^{-10} \mathrm{~m}\right)$. Purge times of $5 \mathrm{~min}$ were used to approach a heliumsaturated steady-state condition. After the wet weights were measured, samples were dried at $110^{\circ} \mathrm{C}$ for $24 \mathrm{hr}$ to drive off water.

The determination of water content followed the methods of the American Society for Testing and Materials (ASTM) designation (D) 2216 (ASTM, 1989). As outlined in the ASTM D2216, corrections are required for salt when measuring marine samples. Samples were saturated in seawater and placed in a vacuum for $24 \mathrm{hr}$ to achieve in-situ wet conditions. All measurements were corrected for salt assuming a pore water salinity of $35 \%$. This method was then used to calculate both bulk density and porosity values.
The bulk density $(\rho)$ is the density of the whole sample, including the pore fluid and is given by

$$
\rho=M_{t} / V_{t},
$$

where:

$M_{t}=$ total mass (saturated) and

$V_{t}=$ saturated sample volume.

The total mass $\left(M_{t}\right)$ was measured using the electronic balance, and the total volume was calculated using the mass of the pore fluid $\left(M_{w}\right)$, the volume of the pore fluid $\left(V_{w}\right)$, and the mass of the dry sample as expressed by the following equations:

$$
M_{w}=\left(M_{t}-M_{d}\right) / s,
$$

where:

$M_{d}=$ dry sample mass,

$s=$ salt factor $=(1-r)=0.965$, and

$r=$ salinity $=0.035$.

This allows the calculation of the pore water volume $\left(V_{w}\right)$,

$$
V_{w}=M_{w} / \rho w,
$$

where:

$\rho w=$ density of pore water $=1.0245 \mathrm{~g} / \mathrm{cm}^{3}$.

The total wet volume $\left(V_{t}\right)$ was then calculated using the following equation:

$$
V_{t}=V_{w}+V_{p}
$$

where:

$V_{d}=$ dry sample volume,

permitting the direct calculation of bulk density values using Equation 1. Overall accuracies in density determinations are about $0.2 \%$ or $0.006 \mathrm{~g} / \mathrm{cm}^{3}$.

The porosity $(\phi)$ was calculated using the expression:

$$
\phi=\left[M_{w} /\left(V_{t} \cdot \rho w\right)\right] \cdot 100 .
$$

These values were determined to approximately $\pm 0.2 \%$, assuming that the porosity is interconnected and fluid saturated.

Temperature effects on seismic velocities also were considered. Using an experimentally derived temperature coefficient $\left(\partial V_{p} / \partial T\right)_{p}$ of $-0.39 \times 10^{-3} \mathrm{~km} / \mathrm{s}^{\circ} \mathrm{C}$ (Christensen, 1979; H. Gröschel-Becker, pers. comm., 1993) and a bottom-hole temperature of approximately $200^{\circ} \mathrm{C}$ (Dick, Erzinger, Stokking, et al., 1992), it was found that the correction for in-situ temperature conditions was negligible.

\section{DISCUSSION}

In the following sections, the influence of porosity, density, alteration, and mineralogical compositions on the seismic properties of Hole 504B are examined. Elastic constants calculated from laboratory velocities and densities are examined in detail along with the elastic behavior of crustal rocks and their significance for future crustal drilling. Comparisons with ophiolite data also are discussed.

\section{Porosity}

As with most oceanic drill holes, fracture porosity is a major factor influencing the velocity structure of Hole 504B. Throughout the core, large populations of open fractures, micro-fractures, and veins have been observed and documented for the samples recovered during Legs 137 and 140 (Dick, Erzinger, Stokking, et al., 1992). These sets 


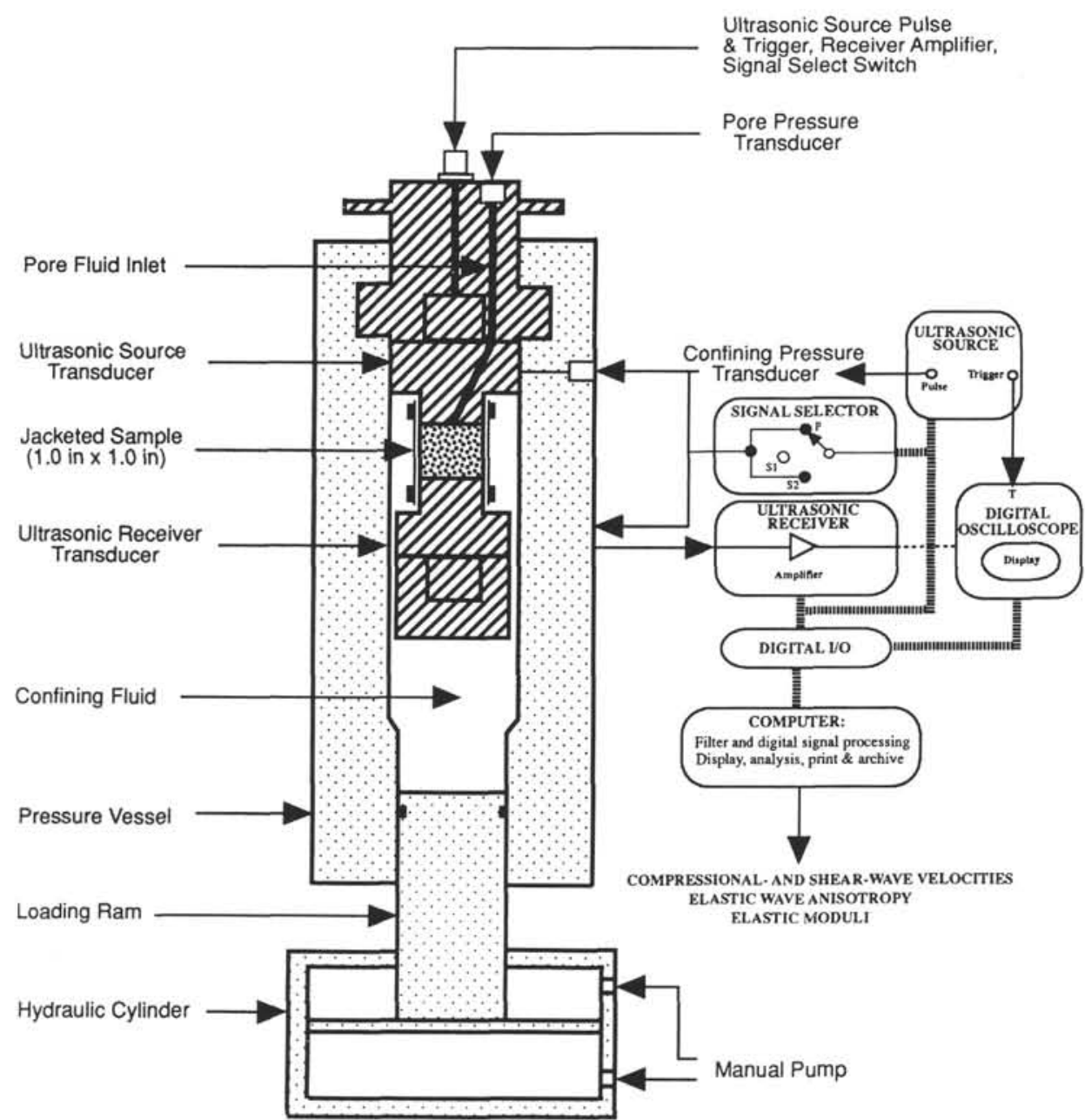

Figure 2. Schematics of the $100 \mathrm{MPa}$ system with the compound ultrasonic transducer system.

of fractures and microcracks may have developed either because of the tectonic setting of the Costa Rica Rift or due to conventional drilling techniques and the unloading of stresses as the samples were brought to the surface. It has been reported previously that open fractures can significantly lower seismic velocities (Birch, 1961, Hyndman and Drury, 1976; Iturrino et al., 1991). The application of confining pressure closes microcracks caused by unloading of in-situ stresses or drilling and diminishes any possible velocity anisotropy created by the presence of oriented fractures. Thus, pressure-velocity measurements can offer better estimates of in-situ velocities at Hole 504B than can atmospheric values measured as part of the shipboard analyses.

Compressional-wave velocities obtained at $100 \mathrm{MPa}$ from previous measurements (Wilkens et al., 1983; Christensen and Salisbury, 1985; Christensen et al., 1989) and cores from Legs 137 and 140 show that seismic velocities decrease with increasing crack porosity for the basement samples of Hole 504B (Fig. 3). At the bottom of the hole, in-situ pressures were calculated to be approximately $90 \mathrm{MPa}$ assuming hydrostatic pressure. This seems to agree with previous estimates of in-situ pressures calculated in ophiolite studies (Salisbury and Christensen, 1978; Christensen and Smewing, 1981; Christensen and Salisbury, 1982). Therefore, $100 \mathrm{MPa}$ pressure was used because this value is close to the in-situ pressures at the bottom of Hole 504B reached during Leg 140. The velocity-porosity plot shows that most of the high porosity and low velocity values were encountered during Legs 69, 70, and 83 (Fig. 3A). Some samples from the lower sections of the hole (Legs 111, 137, and 140) also show high porosities (3\%-
$5 \%$ ), but overall, the velocities cluster around higher velocity and lower porosity values (Fig. 3B) than those from previous legs.

\section{Density and Alteration}

The boundaries between three major regions of alteration obtained from analysis of thin sections in Hole 504B (Alt and Emmermann, 1985; Emmermann, 1985; Alt et al., 1985; 1986; Dick, Erzinger, Stokking, et al., 1992) are shown in Figure 4A. First, this figure shows the contrast in the upper $260 \mathrm{~m}$ between bulk densities and atmospheric pressure velocities of the sediments recovered in Hole 504 and the underlying basement of Hole 504B (Figs. 4A and 4B). The measurements obtained from Hole 504 as part of the shipboard analyses (Cann, Langseth, Honnorez, Von Herzen, White, et al., 1983) were used because this core section was not recovered from Hole 504B and high-pressure velocity measurements were not obtained. Underlying the sedimentary cover, the uppermost basement section, also known as the upper alteration zone, is characterized by reactions between basalt and seawater occurring at temperatures below $60^{\circ} \mathrm{C}$ and by the replacement of olivine throughout the rock by iron hydroxides, celadonite-nontronite, or saponite. The middle section is characterized by suboxic to anoxic alteration reactions at temperatures ranging from $60^{\circ}$ to $110^{\circ} \mathrm{C}$, with the presence of fluids as well as pyrite and saponite as the predominant alteration products. This boundary has been defined previously by geochemical log data and a strong mineral zonation, which suggests a permeability barrier between these two zones (Pezard and Anderson, 1989). This permeability 
Table 1. Compressional- and shear-wave velocities for the samples recovered from Hole 504B during Legs 137 and 140.

\begin{tabular}{|c|c|c|c|c|c|c|c|c|c|c|c|c|c|}
\hline & & & & & & & & & & Velocit & $\operatorname{ty}(\mathrm{km} / \mathrm{s}$ & /s) $-\mathrm{Pr}$ & ressure \\
\hline Sample & $\begin{array}{l}\text { Depth } \\
\text { (mbsf) }\end{array}$ & $\begin{array}{l}\text { Density } \\
\left(\mathrm{g} / \mathrm{cm}^{3}\right)\end{array}$ & $\begin{array}{c}\text { Porosity } \\
\text { (\%) }\end{array}$ & Mode & 10 & 20 & 30 & 40 & 50 & 60 & 70 & 80 & 90 \\
\hline 137-504B-173R-1 (114-116) & 1571.14 & 2.99 & 0.79 & $\mathbf{P}$ & 5.23 & 5.50 & 5.71 & 5.88 & 6.03 & 6.15 & 6.25 & 6.33 & 6.40 \\
\hline & & & & s & 3.16 & 3.28 & 3.37 & 3.44 & 3.51 & 3.56 & 3.60 & 3.63 & 3.66 \\
\hline 137-504B-173R-2 (20-22) & 1571.70 & 2.98 & 0.42 & P & 6.31 & 6.39 & 6.44 & 6.45 & 6.49 & 6.48 & 6.49 & 6.50 & 6.51 \\
\hline & & & & S1 & 3.37 & 3.44 & 3.45 & 3.46 & 3.47 & 3.48 & 3.54 & 3.56 & 3.57 \\
\hline & & & & S2 & 3.39 & 3.43 & 3.44 & 3.46 & 3.48 & 3.49 & 3.54 & 3.56 & 3.57 \\
\hline 137-504B-174R-1 (96-98) & 1577.26 & 2.97 & 0.46 & & & & & & & & & & \\
\hline $137-504 \mathrm{~B}-174 \mathrm{R}-1(116-118)$ & 1577.46 & 2.91 & 0.66 & & & & & & & & & & \\
\hline $137-504 \mathrm{~B}-180 \mathrm{M}-1(94-96)$ & 1619.34 & 2.94 & 0.72 & $\mathbf{P}$ & 6.31 & 6.33 & 6.34 & 6.34 & 6.35 & 6.36 & 6.38 & 6.39 & 6.41 \\
\hline 137-504B-180M-2 & 1620.39 & 2.99 & 0.17 & & & & & & & & & & \\
\hline 137-504B-180M-2 (93-98) & 1620.83 & 3.01 & 0.42 & $\mathrm{P}$ & 5.64 & 5.86 & 6.04 & 6.17 & 6.29 & 6.38 & 6.45 & 6.51 & 6.56 \\
\hline & & & & s & 3.42 & 3.49 & 3.55 & 3.60 & 3.63 & 3.66 & 3.69 & 3.71 & 3.73 \\
\hline 137-504B-181M-2 (148-150) & 1623.38 & 2.91 & 1.87 & P & 6.28 & 6.30 & 6.31 & 6.32 & 6.33 & 6.37 & 6.41 & 6.43 & 6.45 \\
\hline & & & & S1 & 3.43 & 3.43 & 3.45 & 3.46 & 3.46 & 3.48 & 3.50 & 3.51 & 3.51 \\
\hline & & & & S2 & 3.41 & 3.42 & 3.43 & 3.44 & 3.45 & 3.48 & 3.50 & 3.51 & 3.52 \\
\hline $140-504 \mathrm{~B}-186 \mathrm{R}-1(40-42)$ & 1626.70 & 2.88 & 2.02 & $\mathrm{P}$ & 5.80 & 5.82 & 5.91 & 6.00 & 6.08 & 6.17 & 6.24 & 6.31 & 6.34 \\
\hline & & & & S & 3.39 & 3.48 & 3.55 & 3.60 & 3.64 & 3.66 & 3.69 & 3.71 & 3.72 \\
\hline 140-504B-186R-1 (54-56) & 1626.84 & 2.83 & 2.68 & & & & & & & & & & \\
\hline $140-504 \mathrm{~B}-186 \mathrm{R}-1$ & 1627.10 & 2.87 & & & & & & & & & & & \\
\hline $140-50$ & 1627.12 & 2.8 & 1.95 & & & & & & & & & & \\
\hline $140-504 \mathrm{~B}-186 \mathrm{R}-1$ (1 & 1627.51 & 2.91 & 1.51 & P & 6.18 & 6.25 & 6.31 & 6.34 & 6.37 & 6.40 & 6.43 & 6.47 & 6.50 \\
\hline & & & & S1 & 3.43 & 3.49 & 3.56 & 3.60 & 3.61 & 3.63 & 3.69 & 3.74 & 3.76 \\
\hline & & & & S2 & 3.39 & 3.50 & 3.59 & 3.61 & 3.63 & 3.65 & 3.74 & 3.77 & 3.79 \\
\hline $140-504$ & 1628.15 & 2.86 & 2.31 & & & & & & & & & & \\
\hline $140-5$ & 1628.22 & 2.91 & & & & & & & & & & & \\
\hline 140-504B-187R-1 (21-23) & 1632.21 & 2.86 & 2 & & & & & & & & & & \\
\hline$-1(24-27)$ & 1641.74 & 2.96 & 0 & & & & & & & & & & \\
\hline $140-5$ & 1651.96 & 2.9 & 1 & & & & & & & & & & \\
\hline 140-504B-189R-1 (101-103) & 1652.01 & 2.98 & 0.48 & $\mathbf{P}$ & 6.25 & 6.33 & 6.37 & 6.45 & 6.49 & 6.54 & 6.58 & 6.62 & 6.67 \\
\hline & & & & S1 & 3.42 & 3.52 & 3.54 & 3.55 & 3.56 & 3.59 & 3.63 & 3.67 & 3.70 \\
\hline & & & & S2 & 3.39 & 3.45 & 3.48 & 3.51 & 3.54 & 3.57 & 3.62 & 3.66 & 3.69 \\
\hline $0-504$ & 1 & 2.94 & 1 & & & & & & & & & & \\
\hline $140-504 \mathrm{~B}-189 \mathrm{R}-2(72-74)$ & 1653.22 & 2.77 & 2.62 & P & 5.75 & 5.78 & 5.82 & 5.85 & 5.89 & 5.93 & 5.96 & 6.00 & 6.01 \\
\hline & & & & S1 & 3.11 & 3.19 & 3.22 & 3.25 & 3.27 & 3.28 & 3.31 & 3.35 & 3.36 \\
\hline & & & & S2 & 3.14 & 3.22 & 3.27 & 3.30 & 3.32 & 3.33 & 3.37 & 3.39 & 3.40 \\
\hline 140-504B-190R-1 (95-98) & 1656.05 & 276 & & & & & & & & & & & \\
\hline 140-504B-191R-1 (48-50) & 1661.88 & 2.96 & 1.04 & P & 6.20 & 6.32 & 6.36 & 6.39 & 6.41 & 6.44 & 6.46 & 6.51 & 6.59 \\
\hline & & & & S1 & 3.27 & 3.36 & 3.38 & 3.41 & 3.43 & 3.45 & 3.47 & 3.50 & 3.52 \\
\hline & & & & S2 & 3.26 & 3.34 & 3.37 & 3.38 & 3.41 & 3.43 & 3.46 & 3.50 & 3.52 \\
\hline 140-504B-192R-1 (13-14) & 71.03 & 85 & 57 & & & & & & & & & & \\
\hline $140-5$ & 74.57 & & & & & & & & & & & & \\
\hline $140-5$ & 1 & 2. & 4 & & & & & & & & & & \\
\hline $140-504 \mathrm{~B}-194 \mathrm{R}-1(92-95)$ & 1681.32 & 2.79 & 2.75 & $\mathbf{P}$ & 5.35 & 5.54 & 5.67 & 5.75 & 5.82 & 5.87 & 5.91 & 5.94 & 5.96 \\
\hline & & & & s & 3.15 & 3.22 & 3.26 & 3.29 & 3.32 & 3.34 & 3.36 & 3.37 & 3.38 \\
\hline $140-504 \mathrm{~B}-195 \mathrm{R}-1(7-10)$ & 1689.67 & 2.91 & 13 & & & & & & & & & & \\
\hline & & 2. & & & & & & & & & & & \\
\hline $140-504 \mathrm{~B}-197 \mathrm{R}-1(32-34)$ & 1703.12 & 2.95 & 0.85 & $\mathbf{P}$ & 6.25 & 6.30 & 6.35 & 6.39 & 6.40 & 6.42 & 6.46 & 6.50 & 6.52 \\
\hline & & & & S1 & 3.47 & 3.48 & 3.49 & 3.50 & 3.51 & 3.53 & 3.57 & 3.60 & 3.61 \\
\hline & & & & S2 & 3.48 & 3.48 & 3.49 & 3.49 & 3.51 & 3.53 & 3.58 & 3.61 & 3.63 \\
\hline $140-504 \mathrm{~B}-197 \mathrm{R}-1$ (131-134 & 1704.11 & 2.98 & 0.65 & & & & & & & & & & \\
\hline $140-504 \mathrm{~B}-197 \mathrm{R}-1(138-140)$ & 1704.18 & 2.97 & 1.05 & P & 6.32 & 6.38 & 6.44 & 49 & 6.52 & 6.55 & 6.58 & 6.62 & 6.63 \\
\hline & & & & S1 & 3.40 & 3.42 & 3.44 & 3.47 & 3.50 & 3.53 & 3.58 & 3.61 & 3.63 \\
\hline & & & & S2 & 3.33 & 3.38 & 3.42 & 3.45 & 3.49 & 3.51 & 3.58 & 3.62 & 3.65 \\
\hline $140-5$ & 12.90 & 2.89 & 1.13 & & & & & & & & & & \\
\hline $140-504 \mathrm{~B}-199 \mathrm{R}-1$ ( 1 & 1720.61 & 2.99 & 0.47 & $\mathrm{P}$ & 6.08 & 6.25 & 6.33 & 6.34 & 6.36 & 6.38 & 6.40 & 6.41 & 6.43 \\
\hline & & & & S1 & 3.21 & 3.26 & 3.28 & 3.30 & 3.31 & 3.32 & 3.36 & 3.37 & 3.39 \\
\hline & & & & S2 & 3.20 & 3.23 & 3 & 7 & 3.29 & 3.30 & 4 & 5 & 7 \\
\hline $140-504 \mathrm{~B}-200 \mathrm{R}-1(10-12)$ & 1728.70 & 2.99 & 0.31 & $\mathrm{P}$ & 6.17 & 6.27 & 6.32 & 6.39 & 6.42 & 6.47 & 6.49 & 6.52 & 6.55 \\
\hline & & & & S1 & 3.25 & 3.31 & 3.36 & 3.40 & 3.45 & 3.48 & 3.52 & 3.57 & 3.59 \\
\hline & & & & S2 & 3.24 & 3.28 & 3.34 & 3.38 & 3.43 & 3.46 & 3.52 & 3.55 & 3.59 \\
\hline $140-$ & 1729 & & & & & & & & & & & & \\
\hline & & & & & & & & & & & & & \\
\hline $140-504 \mathrm{~B}-200 \mathrm{R}-2(41-43)$ & 1730.52 & 2.98 & 0.91 & $\mathbf{P}$ & 6.24 & 6.35 & 6.41 & 6.43 & 6.48 & 6.54 & 6.55 & 6.57 & 6.61 \\
\hline & & & & S1 & & & & & & & & & \\
\hline & & & & & 3.23 & 3.29 & & 3.42 & & & 3.53 & & 3.60 \\
\hline $140-504 \mathrm{~B}-200 \mathrm{R}-2(44-46)$ & 1730.55 & 2.99 & 0.38 & $\mathrm{P}$ & 4.77 & 5.17 & 5.48 & 5.73 & 5.93 & 6.10 & 6.24 & 6.35 & 6.45 \\
\hline & & & & $\mathrm{s}$ & 2.88 & 3.07 & 3.22 & 3.33 & 3.42 & 3.49 & 3.55 & 3.59 & 3.63 \\
\hline $140-504 \mathrm{~B}-200 \mathrm{R}-2(73-75)$ & 1730.84 & 2.97 & 0.34 & $\mathrm{P}$ & 6.28 & 6.37 & 6.44 & & 6.50 & 6.51 & 6.56 & & 6.61 \\
\hline & & & & S1 & 3.32 & 3.34 & 3.38 & 3.41 & & 3.45 & 3.50 & 3.54 & 3.56 \\
\hline & & & & S2 & 3.24 & 3.32 & 3.35 & 3.39 & 3.41 & 3.44 & 3.48 & 3.53 & 3.55 \\
\hline $140-5$ & 31.51 & & 0 & & & & & & & & & & \\
\hline & & & & & & & & & & & & & \\
\hline & 1 & & & & & & & & & & & & \\
\hline & & & & & & & & & & & & & \\
\hline & i) & 2 . & & & & & & & & & & & \\
\hline $140-504 \mathrm{~B}-203 \mathrm{R}-1(57-59)$ & 1749.57 & 2.90 & 1.53 & $\mathrm{P}$ & & 6.36 & & & 6.44 & & & & \\
\hline & & & & & 3.44 & 3.47 & 3.49 & 3.50 & 3.51 & 3.52 & 3.56 & 3.58 & 3.58 \\
\hline & & & & 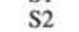 & 3.44 & 3.49 & 3.51 & 3.52 & 3.52 & 3.53 & 3.56 & 3.58 & 3.59 \\
\hline & & 2. & & & & & & & & & & & \\
\hline & 1757.03 & 2.91 & 1.58 & & 6.40 & 6.44 & 6.47 & 6.48 & 6.50 & 6.52 & 6.54 & 6.56 & 6.57 \\
\hline & & & & S1 & 3.48 & 3.49 & 3.49 & 3.51 & 3.51 & 3.52 & 3.53 & 3.54 & 3.55 \\
\hline & & & & S2 & 3.48 & 3.49 & 3.49 & 3.49 & 3.50 & 3.51 & 3.52 & 3.55 & 3.55 \\
\hline $\begin{array}{l}140-504 \mathrm{~B}-205 \\
140-504 \mathrm{~B}-206\end{array}$ & & & & & & & & & & & & & \\
\hline $140-504 \mathrm{~B}-207 \mathrm{R}-1(4-6)$ & 1768.44 & 2.98 & 1.05 & $\mathrm{P}$ & 5.15 & 5.48 & 5.54 & 5.60 & 5.74 & 5.86 & 5.99 & 6.13 & 6.23 \\
\hline
\end{tabular}


Table 1 (continued).

\begin{tabular}{|c|c|c|c|c|c|c|c|c|c|c|c|c|c|c|c|c|c|c|}
\hline & & & & & & & & & & Velocit & $\mathrm{y}(\mathrm{km} /$ & (s) $-\operatorname{Pr}$ & ressure & $(\mathrm{MPa})$ & & & & \\
\hline Sample & $\begin{array}{l}\text { Depth } \\
\text { (mbsf) }\end{array}$ & $\begin{array}{l}\text { Density } \\
\left(\mathrm{g} / \mathrm{cm}^{3}\right)\end{array}$ & $\begin{array}{c}\text { Porosity } \\
(\%)\end{array}$ & Mode & 10 & 20 & 30 & 40 & 50 & 60 & 70 & 80 & 90 & 100 & 200 & 300 & 400 & 500 \\
\hline & & & & $S$ & 3.32 & 3.38 & 3.41 & 3.44 & 3.48 & 3.52 & 3.57 & 3.62 & 3.65 & 3.68 & 3.81 & 3.84 & 3.85 & 3.85 \\
\hline $140-504 \mathrm{~B}-207 \mathrm{R}-1(20-23)$ & 1768.60 & 2.94 & $\begin{array}{l}0.79 \\
0.54\end{array}$ & & & & & & & & & & & & & & & \\
\hline 140-504B-208R-1 (4-7) & 1778.04 & 2.98 & 0.54 & $P$ & 4.98 & 5.12 & 5.24 & 5.36 & 5.52 & 5.68 & 5.84 & 5.98 & 6.11 & 6.24 & 6.84 & 6.98 & 7.03 & 7.06 \\
\hline 140-504B-208R-1 (85-87) & 1778.85 & 2.88 & 1.51 & $\begin{array}{l}\mathrm{S} \\
\mathrm{P} \\
\mathrm{S} 1 \\
\mathrm{~S} 2\end{array}$ & $\begin{array}{l}3.09 \\
6.06 \\
3.30 \\
3.33\end{array}$ & $\begin{array}{l}3.13 \\
6.09 \\
3.34 \\
3.35\end{array}$ & $\begin{array}{l}3.20 \\
6.13 \\
3.34 \\
3.36\end{array}$ & $\begin{array}{l}3.27 \\
6.15 \\
3.35 \\
3.39\end{array}$ & $\begin{array}{l}3.33 \\
6.17 \\
3.36 \\
3.39\end{array}$ & $\begin{array}{l}3.39 \\
6.19 \\
3.37 \\
3.40\end{array}$ & $\begin{array}{l}3.44 \\
6.22 \\
3.39 \\
3.41\end{array}$ & $\begin{array}{l}3.50 \\
6.23 \\
3.39 \\
3.42\end{array}$ & $\begin{array}{l}3.54 \\
6.25 \\
3.40 \\
3.42\end{array}$ & $\begin{array}{l}3.57 \\
6.27 \\
3.41 \\
3.44\end{array}$ & 3.75 & 3.80 & 3.81 & 3.82 \\
\hline 140-504B-208R-1 (132-134) & 1779.18 & 2.97 & 1.12 & $\begin{array}{l}P \\
S 1 \\
S 2\end{array}$ & $\begin{array}{l}6.02 \\
3.22 \\
3.18\end{array}$ & $\begin{array}{l}6.18 \\
3.26 \\
3.25\end{array}$ & $\begin{array}{l}6.26 \\
3.32 \\
3.31\end{array}$ & $\begin{array}{l}6.34 \\
3.39 \\
3.37\end{array}$ & $\begin{array}{l}6.40 \\
3.43 \\
3.42\end{array}$ & $\begin{array}{l}6.45 \\
3.48 \\
3.47\end{array}$ & $\begin{array}{l}6.49 \\
3.53 \\
3.52\end{array}$ & $\begin{array}{l}6.55 \\
3.59 \\
3.59\end{array}$ & $\begin{array}{l}6.58 \\
3.61 \\
3.61\end{array}$ & $\begin{array}{l}6.60 \\
3.65 \\
3.65\end{array}$ & & & & \\
\hline 140-504B-208R-2(10-12) & 1779.60 & 2.99 & 0.38 & $\begin{array}{l}\mathrm{S} 2 \\
\mathrm{P}\end{array}$ & $\begin{array}{l}3.18 \\
6.16\end{array}$ & $\begin{array}{l}3.25 \\
6.24\end{array}$ & $\begin{array}{l}3.31 \\
6.31\end{array}$ & $\begin{array}{l}3.37 \\
6.38\end{array}$ & $\begin{array}{l}3.42 \\
6.40\end{array}$ & $\begin{array}{l}3.47 \\
6.44\end{array}$ & $\begin{array}{l}3.52 \\
6.47\end{array}$ & $\begin{array}{l}3.59 \\
6.54\end{array}$ & $\begin{array}{l}3.61 \\
6.56\end{array}$ & $\begin{array}{l}3.65 \\
6.61\end{array}$ & & & & \\
\hline & & & & S1 & 3.38 & 3.43 & 3.49 & 3.51 & 3.53 & 3.54 & 3.59 & 3.62 & 3.65 & 3.67 & & & & \\
\hline & & & & S2 & 3.37 & 3.43 & 3.48 & 3.50 & 3.54 & 3.56 & 3.62 & 3.64 & 3.67 & 3.69 & & & & \\
\hline 140-504B-208R-3 (6-8) & 1781.06 & 2.75 & 3.44 & P & 5.68 & $\begin{array}{l}5.74 \\
3.38\end{array}$ & $\begin{array}{l}5.78 \\
3.39\end{array}$ & $\begin{array}{l}5.83 \\
3.41\end{array}$ & $\begin{array}{l}5.86 \\
3.41\end{array}$ & $\begin{array}{l}5.90 \\
3.42\end{array}$ & $\begin{array}{l}5.91 \\
3.43\end{array}$ & $\begin{array}{l}5.93 \\
3.47\end{array}$ & $\begin{array}{l}5.95 \\
3.48\end{array}$ & $\begin{array}{l}5.97 \\
3.50\end{array}$ & & & & \\
\hline & & & & $\begin{array}{l}\text { S1 } \\
\text { S2 }\end{array}$ & $\begin{array}{l}3.33 \\
3.33\end{array}$ & $\begin{array}{l}3.38 \\
3.34\end{array}$ & $\begin{array}{l}3.39 \\
3.35\end{array}$ & $\begin{array}{l}3.41 \\
3.37\end{array}$ & $\begin{array}{l}3.41 \\
3.38\end{array}$ & $\begin{array}{l}3.42 \\
3.39\end{array}$ & $\begin{array}{l}3.43 \\
3.42\end{array}$ & $\begin{array}{l}3.47 \\
3.46\end{array}$ & $\begin{array}{l}3.48 \\
3.47\end{array}$ & $\begin{array}{l}3.50 \\
3.49\end{array}$ & & & & \\
\hline $140-504 \mathrm{~B}-209 \mathrm{R}-1(29-31)$ & 1787.79 & 2.92 & 0.39 & & & & & & & & & & & & & & & \\
\hline 140-504B-209R-1 (103-105) & 1788.53 & 2.77 & 4.23 & $\mathrm{P}$ & 5.54 & 5.76 & 5.91 & 6.01 & 6.08 & 6.13 & 6.17 & 6.20 & 6.22 & 6.23 & 6.31 & 6.35 & 6.38 & 6.40 \\
\hline & & & & $\mathrm{S}$ & 3.26 & 3.34 & 3.38 & 3.40 & 3.42 & 3.44 & 3.45 & 3.46 & 3.47 & 3.48 & 3.56 & 3.62 & 3.67 & 3.70 \\
\hline 140-504B-209R-2 (5-7) & 1789.05 & 2.89 & 1.26 & $\mathrm{P}$ & 5.87 & 5.97 & 6.02 & 6.08 & 6.13 & 6.19 & 6.25 & 6.30 & 6.36 & 6.42 & & & & \\
\hline & & & & S1 & 3.12 & 3.26 & 3.29 & 3.34 & 3.37 & 3.41 & 3.48 & 3.51 & 3.53 & 3.57 & & & & \\
\hline & & & & S2 & 3.12 & 3.25 & 3.32 & 3.35 & 3.40 & 3.42 & 3.49 & 3.52 & 3.56 & 3.58 & & & & \\
\hline 140-504B-209R-2 (9-11) & 1789.09 & 2.81 & 3.30 & & & & & & & & & & & & & & & \\
\hline 140-504B-209R-2 (124-126) & 1790.24 & 2.92 & 1.43 & $\mathrm{P}$ & 6.30 & 6.34 & 6.38 & 6.40 & 6.42 & 6.44 & 6.47 & 6.51 & 6.53 & 6.56 & & & & \\
\hline & & & & S1 & 3.30 & 3.36 & 3.41 & 3.44 & 3.46 & 3.49 & 3.52 & 3.59 & 3.60 & 3.61 & & & & \\
\hline & & & & $\mathrm{S} 2$ & 3.33 & 3.38 & 3.41 & 3.44 & 3.47 & 3.50 & 3.55 & 3.60 & 3.62 & 3.63 & & & & \\
\hline 140-504B-210R-1 (18-20) & 1795.08 & 2.98 & 1.04 & & & & & & & & & & & & & & & \\
\hline 140-504B-210R-1 (27-29) & 1795.17 & 2.99 & 0.34 & P & 6.02 & 6.16 & 6.26 & 6.34 & 6.38 & 6.46 & 6.54 & 6.62 & 6.66 & 6.70 & & & & \\
\hline & & & & S1 & 3.07 & 3.18 & 3.28 & 3.35 & 3.42 & 3.46 & 3.55 & 3.60 & 3.64 & 3.66 & & & & \\
\hline & & & & S2 & 3.11 & 3.21 & 3.31 & 3.38 & 3.44 & 3.49 & 3.57 & 3.63 & 3.67 & 3.68 & & & & \\
\hline 140-504B-210R-1 (117-119) & 1796.07 & 2.96 & 1.31 & & & & & & & & & & & & & & & \\
\hline 140-504B-210R-2 (2-4) & 1796.42 & 3.00 & 0.23 & P & 6.24 & 6.32 & 6.40 & 6.46 & 6.50 & 6.54 & 6.57 & 6.61 & 6.63 & 6.65 & & & & \\
\hline & & & & S1 & 3.43 & 3.46 & 3.48 & 3.50 & 3.51 & 3.54 & 3.58 & 3.64 & 3.66 & 3.69 & & & & \\
\hline & & & & $\mathrm{S} 2$ & 3.44 & 3.45 & 3.49 & 3.52 & 3.53 & 3.55 & 3.60 & 3.66 & 3.68 & 3.71 & & & & \\
\hline 140-504B-210R-2 (5-7) & 1796.45 & 2.98 & 1.38 & & & & & & & & & & & & & & & \\
\hline $140-504 B-211 R-1(14-16)$ & 1798.64 & 2.96 & 0.86 & $\mathrm{P}$ & 6.40 & 6.45 & 6.47 & 6.53 & 6.55 & 6.57 & 6.59 & 6.61 & 6.63 & 6.65 & & & & \\
\hline & & & & S1 & 3.42 & 3.46 & 3.50 & 3.53 & 3.55 & 3.57 & 3.61 & 3.66 & 3.69 & 3.71 & & & & \\
\hline & & & & S2 & 3.41 & 3.45 & 3.49 & 3.53 & 3.55 & 3.58 & 3.63 & 3.68 & 3.71 & 3.73 & & & & \\
\hline $140-504 B-212 R-1(4-7)$ & 1806.04 & 2.98 & 0.57 & & & & & & & & & & & & & & & \\
\hline $140-504 \mathrm{~B}-212 \mathrm{R}-1(44-46)$ & 1806.44 & 2.92 & 1.49 & & & & & & & & & & & & & & & \\
\hline $140-504 B-213 R-1$ (49-51) & 1812.99 & 2.91 & 1.48 & $P$ & 6.26 & 6.32 & 6.38 & 6.40 & 6.41 & 6.47 & 6.50 & 6.53 & 6.55 & 6.57 & & & & \\
\hline & & & & S1 & 3.30 & 3.37 & 3.40 & 3.44 & 3.45 & 3.46 & 3.50 & 3.52 & 3.53 & 3.54 & & & & \\
\hline & & & & S2 & 3.27 & 3.34 & 3.38 & 3.42 & 3.44 & 3.46 & 3.50 & 3.51 & 3.52 & 3.54 & & & & \\
\hline $140-504 B-214 R-1(31-33)$ & 1818.91 & 2.91 & 1.47 & $P$ & 5.88 & 5.99 & 6.01 & 6.09 & 6.12 & 6.14 & 6.19 & 6.21 & 6.23 & 6.25 & & & & \\
\hline & & & & S1 & 3.23 & 3.30 & 3.32 & 3.34 & 3.35 & 3.36 & 3.38 & 3.38 & 3.39 & 3.39 & & & & \\
\hline & & & & S2 & 3.38 & 3.39 & 3.41 & 3.42 & 3.42 & 3.42 & 3.43 & 3.44 & 3.45 & 3.46 & & & & \\
\hline $140-504 \mathrm{~B}-214 \mathrm{R}-1(44-46)$ & 1819.04 & 2.91 & 0.95 & P & 6.17 & 6.25 & 6.28 & 6.34 & 6.36 & 6.40 & 6.41 & 6.45 & 6.47 & 6.49 & & & & \\
\hline & & & & S1 & 3.29 & 3.33 & 3.38 & 3.42 & 3.45 & 3.49 & 3.52 & 3.57 & 3.60 & 3.62 & & & & \\
\hline & & & & S2 & 3.33 & 3.37 & 3.41 & 3.45 & 3.48 & 3.52 & 3.56 & 3.62 & 3.65 & 3.66 & & & & \\
\hline $140-504 B-214 R-2(88-90)$ & 1820.98 & 2.96 & 0.87 & P & 6.30 & 6.37 & 6.46 & 6.48 & 6.51 & 6.53 & 6.57 & 6.59 & 6.63 & 6.65 & & & & \\
\hline & & & & S1 & 3.40 & 3.47 & 3.49 & 3.51 & 3.52 & 3.53 & 3.56 & 3.58 & 3.59 & 3.60 & & & & \\
\hline & & & & $\mathrm{S} 2$ & 3.35 & 3.44 & 3.49 & 3.52 & 3.53 & 3.54 & 3.57 & 3.58 & 3.60 & 3.60 & & & & \\
\hline 140-504B-214R-2 (130-132) & 1821.40 & 2.79 & 3.28 & & & & & & & & & & & & & & & \\
\hline 140-504B-215R-1 (1-4) & 1823.01 & 2.97 & 0.67 & & & & & & & & & & & & & & & \\
\hline 140-504B-216R-1 (58-60) & 1828.48 & 2.89 & 1.88 & & & & & & & & & & & & & & & \\
\hline $140-540 \mathrm{~B}-217 \mathrm{R}-1(4-6)$ & 1837.44 & 2.84 & 2.96 & & & & & & & & & & & & & & & \\
\hline 140-504B-218R-1 (24-27) & 1847.14 & 2.90 & 1.33 & & & & & & & & & & & & & & & \\
\hline 140-504B-219R-1 (7-11) & 1856.27 & 2.98 & 0.36 & & & & & & & & & & & & & & & \\
\hline 140-504B-220R-1 (2-6) & 1865.52 & 2.91 & 0.75 & & & & & & & & & & & & & & & \\
\hline 140-504B-220R-1 (27-30) & 1865.77 & 2.93 & 1.08 & & & & & & & & & & & & & & & \\
\hline 140-504B-221R-1 (4-7) & 1875.04 & 2.97 & 0.72 & & & & & & & & & & & & & & & \\
\hline 140-504B-221R-1 (44 46) & 1875.44 & 2.91 & 1.20 & & & & & & & & & & & & & & & \\
\hline $140-504 \mathrm{~B}-222 \mathrm{R}-1(17-19)$ & 1884.77 & 2.88 & 1.38 & $P$ & 6.17 & 6.23 & 6.27 & 6.30 & 6.32 & 6.35 & 6.37 & 6.40 & 6.42 & 6.45 & & & & \\
\hline & & & & S1 & 3.32 & 3.34 & 3.35 & 3.36 & 3.37 & 3.37 & 3.40 & 3.41 & 3.42 & 3.42 & & & & \\
\hline & & & & S2 & 3.30 & 3.34 & 3.36 & 3.36 & 3.37 & 3.39 & 3.42 & 3.42 & 3.44 & 3.45 & & & & \\
\hline 140-504B-222R-1 (37-39) & 1884.97 & 2.89 & 1.33 & & & & & & & & & & & & & & & \\
\hline 140-504B-222R-1 (100-104) & 1885.60 & 2.94 & 0.94 & & & & & & & & & & & & & & & \\
\hline 140-504B-222R-1 (129-132) & 1885.89 & 2.83 & 2.00 & & & & & & & & & & & & & & & \\
\hline $140-504 \mathrm{~B}-223 \mathrm{R}-1(20-23)$ & 1894.44 & 2.97 & 0.78 & & & & & & & & & & & & & & & \\
\hline $140-504 \mathrm{~B}-224 \mathrm{R}-1$ (59-62) & 1904.29 & 2.95 & 1.03 & & & & & & & & & & & & & & & \\
\hline 140-504B-225R-1 (28-30) & 1912.48 & 2.95 & 0.96 & & & & & & & & & & & & & & & \\
\hline 140-504B-225R-1 (137-139) & 1913.57 & 2.98 & 0.39 & & & & & & & & & & & & & & & \\
\hline $140-504 B-225 R-2(14-16)$ & 1913.84 & 2.98 & 1.20 & $\mathrm{P}$ & 6.15 & 6.25 & 6.34 & 6.38 & 6.40 & 6.45 & 6.49 & 6.54 & 6.56 & 6.58 & & & & \\
\hline & & & & S1 & 3.14 & 3.26 & 3.34 & 3.39 & 3.43 & 3.47 & 3.53 & 3.57 & 3.60 & 3.61 & & & & \\
\hline & & & & S2 & 3.16 & 3.25 & 3.33 & 3.37 & 3.43 & 3.46 & 3.54 & 3.57 & 3.60 & 3.62 & & & & \\
\hline $140-504 B-225 R-2(42-44)$ & 1914.12 & 2.92 & 0.20 & & & & & & & & & & & & & & & \\
\hline $140-504 \mathrm{~B}-225 \mathrm{R}-2(105-107)$ & 1914.75 & 2.96 & 1.00 & $\mathrm{P}$ & 5.89 & 6.11 & 6.25 & 6.33 & 6.40 & 6.44 & 6.50 & 6.57 & 6.59 & 6.62 & & & & \\
\hline & & & & S1 & 3.15 & 3.26 & 3.35 & 3.41 & 3.46 & 3.50 & 3.59 & 3.64 & 3.67 & 3.69 & & & & \\
\hline & & & & S2 & 3.13 & 3.24 & 3.33 & 3.40 & 3.46 & 3.50 & 3.58 & 3.63 & 3.66 & 3.68 & & & & \\
\hline 140-504B-226R-1 (61-63) & 1920.61 & 2.94 & 0.78 & $\mathbf{P}$ & 6.20 & 6.29 & 6.34 & 6.38 & 6.40 & 6.43 & 6.45 & 6.46 & 6.48 & 6.49 & 6.58 & 6.63 & 6.67 & 6.71 \\
\hline & & & & $\mathrm{S}$ & 3.04 & 3.20 & 3.32 & 3.42 & 3.50 & 3.56 & 3.60 & 3.64 & 3.67 & 3.69 & 3.78 & 3.80 & 3.81 & 3.82 \\
\hline 140-504B-226R-1 (144-146) & 1921.44 & 2.94 & 0.48 & $\mathbf{P}$ & 6.21 & 6.27 & 6.32 & 6.35 & 6.37 & 6.40 & 6.43 & 6.49 & 6.51 & 6.54 & & & & \\
\hline & & & & S1 & 3.43 & 3.48 & 3.50 & 3.52 & 3.54 & 3.54 & 3.57 & 3.62 & 3.65 & 3.67 & & & & \\
\hline & & & & S2 & 3.44 & 3.49 & 3.52 & 3.54 & 3.54 & 3.57 & 3.60 & 3.64 & 3.66 & 3.68 & & & & \\
\hline
\end{tabular}


Table 1 (continued).

\begin{tabular}{|c|c|c|c|c|c|c|c|c|c|c|c|c|c|c|c|c|c|c|c|}
\hline \multirow[b]{2}{*}{ Sample } & \multirow[b]{2}{*}{$\begin{array}{l}\text { Depth } \\
\text { (mbsf) }\end{array}$} & \multirow[b]{2}{*}{$\begin{array}{l}\text { Density } \\
\left(\mathrm{g} / \mathrm{cm}^{3}\right)\end{array}$} & \multirow[b]{2}{*}{$\begin{array}{c}\text { Porosity } \\
(\%)\end{array}$} & \multirow[b]{2}{*}{ Mode } & \multicolumn{15}{|c|}{ Velocity $(\mathrm{km} / \mathrm{s})$ - Pressure $(\mathrm{MPa})$} \\
\hline & & & & & 10 & 20 & 30 & 40 & 50 & 60 & 70 & 80 & 90 & 100 & 200 & 300 & 400 & 500 & 600 \\
\hline \multirow[t]{3}{*}{$140-504 \mathrm{~B}-226 \mathrm{R}-2(66-68)$} & 1922.16 & 2.88 & 1.88 & P & 6.01 & 6.09 & 6.15 & 6.21 & 6.25 & 6.27 & 6.29 & 6.31 & 6.33 & 6.35 & & & & & \\
\hline & & & & S1 & 3.46 & 3.48 & 3.50 & 3.54 & 3.57 & 3.60 & 3.64 & 3.69 & 3.72 & 3.72 & & & & & \\
\hline & & & & S2 & 3.48 & 3.50 & 3.51 & 3.55 & 3.58 & 3.62 & 3.66 & 3.70 & 3.72 & 3.73 & & & & & \\
\hline \multirow[t]{2}{*}{$140-504 \mathrm{~B}-226 \mathrm{R}-2(77-79)$} & 1922.27 & 2.95 & 0.57 & $\mathrm{P}$ & 4.86 & 5.05 & 5.20 & 5.35 & 5.53 & 5.71 & 5.85 & 5.99 & 6.09 & 6.19 & 6.66 & 6.77 & 6.80 & 6.84 & 6.87 \\
\hline & & & & S & 2.94 & 3.09 & 3.19 & 3.28 & 3.34 & 3.40 & 3.45 & 3.50 & 3.54 & 3.57 & 3.75 & 3.79 & 3.80 & 3.81 & 3.82 \\
\hline \multirow[t]{3}{*}{$140-504 \mathrm{~B}-226 \mathrm{R}-2(120-122)$} & 1922.70 & 2.97 & 0.21 & $\mathrm{P}$ & 6.06 & 6.19 & 6.32 & 6.36 & 6.42 & 6.44 & 6.46 & 6.51 & 6.53 & 6.55 & & & & & \\
\hline & & & & S1 1 & 3.17 & 3.25 & 3.37 & 3.40 & 3.44 & 3.48 & 3.54 & 3.58 & 3.61 & 3.63 & & & & & \\
\hline & & & & $\mathrm{S} 2$ & 3.19 & 3.29 & 3.39 & 3.45 & 3.48 & 3.51 & 3.56 & 3.60 & 3.62 & 3.64 & & & & & \\
\hline $140-504 \mathrm{~B}-226 \mathrm{R}-2(130-132)$ & 1922.80 & 2.90 & 2.80 & & & & & & & & & & & & & & & & \\
\hline $140-504 \mathrm{~B}-226 \mathrm{R}-3(2-4)$ & 1923.02 & 2.94 & 0.72 & & & & & & & & & & & & & & & & \\
\hline \multirow[t]{2}{*}{$140-504 \mathrm{~B}-227 \mathrm{R}-1$ (83-85) } & 1925.33 & 2.96 & 0.46 & $P$ & 4.58 & 4.82 & 5.03 & 5.23 & 5.43 & 5.63 & 5.79 & 5.95 & 6.04 & 6.13 & 6.73 & 6.90 & 6.95 & 7.00 & 7.01 \\
\hline & & & & S & 2.76 & 2.93 & 3.03 & 3.12 & 3.22 & 3.33 & 3.39 & 3.45 & 3.50 & 3.55 & 3.76 & 3.81 & 3.82 & 3.83 & 3.84 \\
\hline $140-504 \mathrm{~B}-227 \mathrm{R}-2(10-12)$ & 1926.10 & 2.82 & 2.49 & & & & & & & & & & & & & & & & \\
\hline \multirow[t]{2}{*}{$140-504 \mathrm{~B}-228 \mathrm{R}-1$ (55-57) } & 1934.55 & 2.97 & 0.55 & $\mathrm{P}$ & 5.57 & 5.92 & 6.16 & 6.33 & 6.45 & 6.54 & 6.60 & 6.65 & 6.69 & 6.72 & 6.82 & 6.86 & 6.89 & 6.91 & 6.93 \\
\hline & & & & S & 3.15 & 3.28 & 3.39 & 3.46 & 3.52 & 3.57 & 3.60 & 3.63 & 3.66 & 3.67 & 3.75 & 3.77 & 3.79 & 3.80 & 3.81 \\
\hline \multirow[t]{3}{*}{$140-504 B-236 R-1(32-34)$} & 1981.02 & 2.87 & 2.96 & $\mathbf{P}$ & 6.16 & 6.20 & 6.25 & 6.29 & 6.33 & 6.38 & 6.40 & 6.45 & 6.47 & 6.49 & & & & & \\
\hline & & & & & S1 & 3.41 & 3.52 & 3.55 & 3.56 & 3.57 & 3.59 & 3.59 & 3.60 & 3.61 & 3.62 & & & & \\
\hline & & & & & S2 & 3.44 & 3.55 & 3.57 & 3.59 & 3.61 & 3.62 & 3.62 & 3.62 & 3.64 & 3.65 & & & & \\
\hline \multirow{3}{*}{$140-504 \mathrm{~B}-236 \mathrm{R}-1$ (53-55) } & 1981.23 & 2.93 & 0.40 & P & 6.12 & 6.21 & 6.30 & 6.36 & 6.39 & 6.42 & 6.45 & 6.52 & 6.55 & 6.58 & & & & & \\
\hline & & & & & S1 & 3.22 & 3.30 & 3.36 & 3.41 & 3.46 & 3.49 & 3.53 & 3.60 & 3.64 & 3.66 & & & & \\
\hline & & & & & S2 & 3.18 & 3.27 & 3.34 & 3.39 & 3.42 & 3.46 & 3.51 & 3.58 & 3.61 & 3.64 & & & & \\
\hline $140-504 \mathrm{~B}-238 \mathrm{R}-1(23-25)$ & 1992.33 & 2.99 & 0.38 & & & & & & & & & & & & & & & & \\
\hline
\end{tabular}

Notes: $\mathrm{P}=$ compressional-wave velocities; $\mathrm{S}=$ shear-wave velocities. In cases where two orthogonally polarized shear-waves were measured, $\mathrm{S} 1$ represents a particle motion in a horizontal plane with respect to the drill-core, and S2 vibrates in a vertical plane or parallel to the borehole.
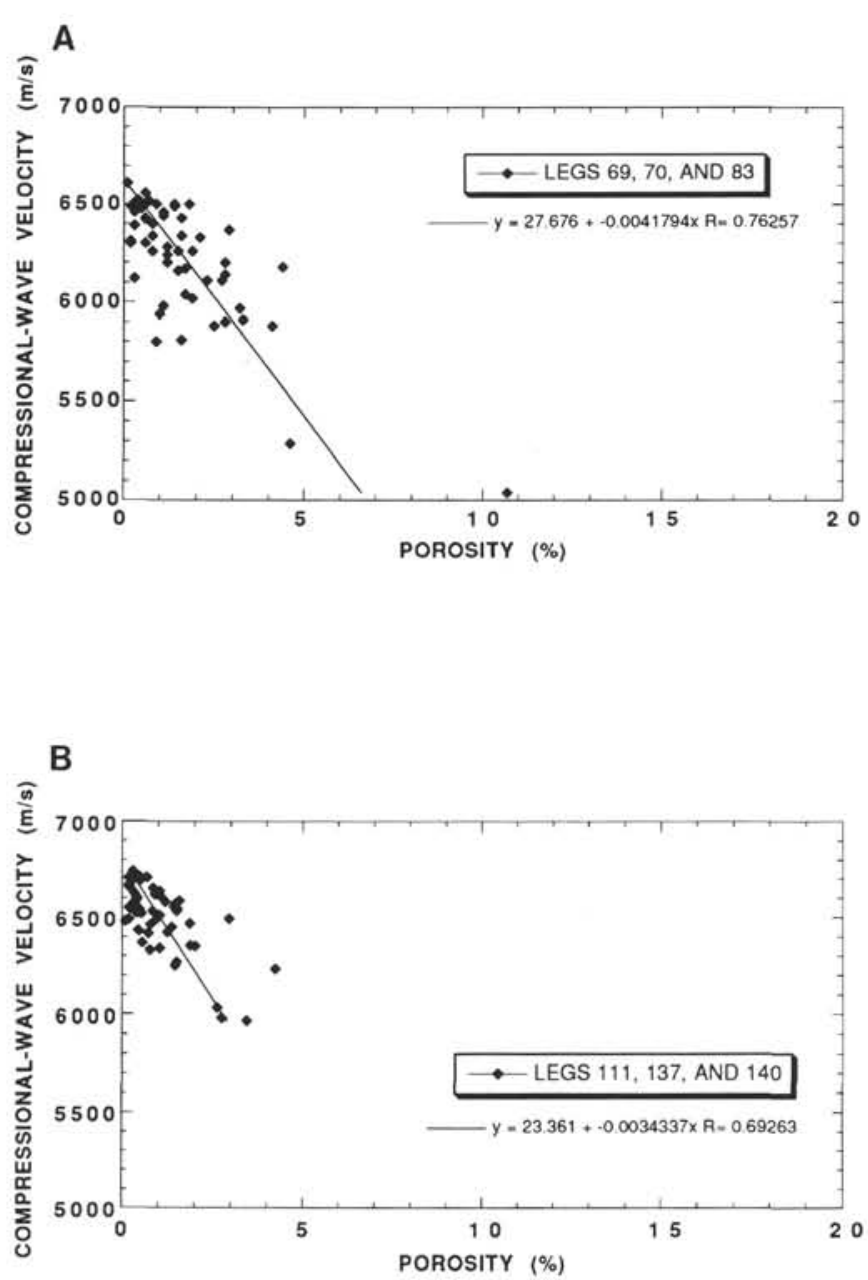

Figure 3. Effect of crack porosity on compressional-wave velocities throughout Hole 504B with velocities measured at $100 \mathrm{MPa}$. A. Samples from Legs 69 , 70, and 83. B. Samples from Legs 111, 137, and 140. barrier is shown in the shallow laterolog (Fig. 5) as well as in porosity, density, and velocity profiles displayed as functions of depth (Figs. $4 \mathrm{~A}$ and $4 \mathrm{~B}$ ). The shallow laterolog (LLS) is sensitive to both the horizontal and vertical resistivity of the rock (Pezard and Anderson, 1989) and is consequently affected by pore fluid conduction within the fractures separating the two distinct hydrothermal regimes. Finally, the transition between anoxic (or suboxic) alteration and greenschist facies alteration is characterized by the recrystallization of clinopyroxenes to actinolite; the replacement of olivine by chlorite, mixedlayer clays, talc, pyrite, and minor actinolite; the variable alteration of plagioclase to albite, chlorite, mixed-layer clays and calcic zeolites; and the replacements of titanomagnetite by titanite.

Density vs. compressional-wave velocity plots for horizontal cores recovered during Legs 137 and 140 show some interesting aspects of the sheeted dikes section drilled at this site. First, velocities measured at $600 \mathrm{MPa}$ were used to examine the effects of mineralogical composition and to minimize the effects of crack porosity. This diagram shows a well-defined linear trend of decreasing velocities with decreasing density (Fig. 6A) as well as some of the highest bulk densities and compressional-wave velocities measured in Hole 504B. With the lack of $1000 \mathrm{MPa}$ data and chemical analyses, lines of constant mean atomic weight at $1000 \mathrm{MPa}$ were obtained from Birch (1961), corrected for $600 \mathrm{MPa}$ pressures (Table 2), and plotted on the velocitydensity diagram to emphasize the significance of chemical composition on seismic velocities. Birch's law is an empirically derived relationship used for solid aggregates in which a linear relationship between density and velocity is assumed and the effects of cracks are minimal. Depending on the porosity, the confining pressure vs. velocity relationship begins a linear trend around a range of $100-\mathrm{MPa}$ to 200-MPa pressure values (Christensen et al., 1989), and based on hysteresis recorded in laboratory samples, the effects of cracks in low porosity samples begin to become negligible around the same values (Christensen et al., 1989; Iturrino et al., 1991). Hysteresis in laboratory measurements reflects the closure and opening of fractures with increasing and decreasing confining pressure, respectively, with higher velocity values usually recorded during the downgoing pressure steps because the cracks do not open as quickly and as far during pressure reduction. Therefore, the use of $600-\mathrm{MPa}$ velocity values for a comparison with mean atomic weights seems valid.

Iron-free silicates have mean atomic weights of near 20 , and the reported mean atomic weight for most common rocks is 21 (Birch, 1961). The most common elements producing deviations from 20 are 


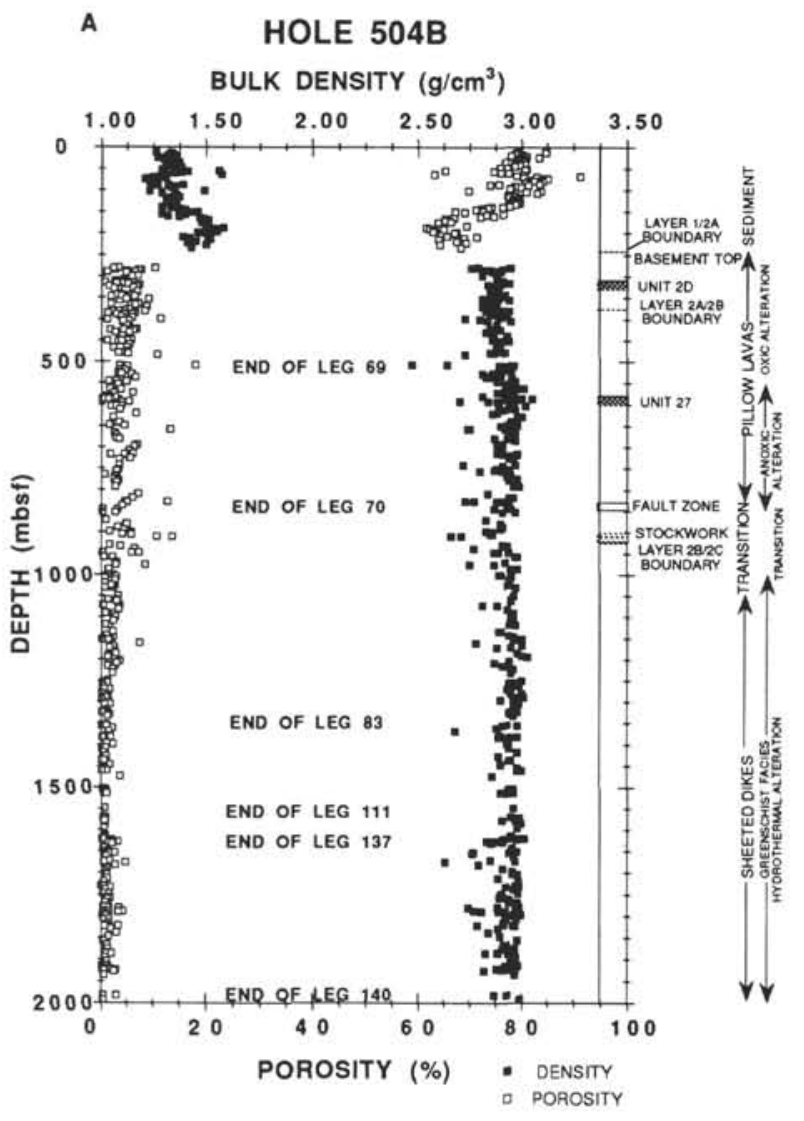

B

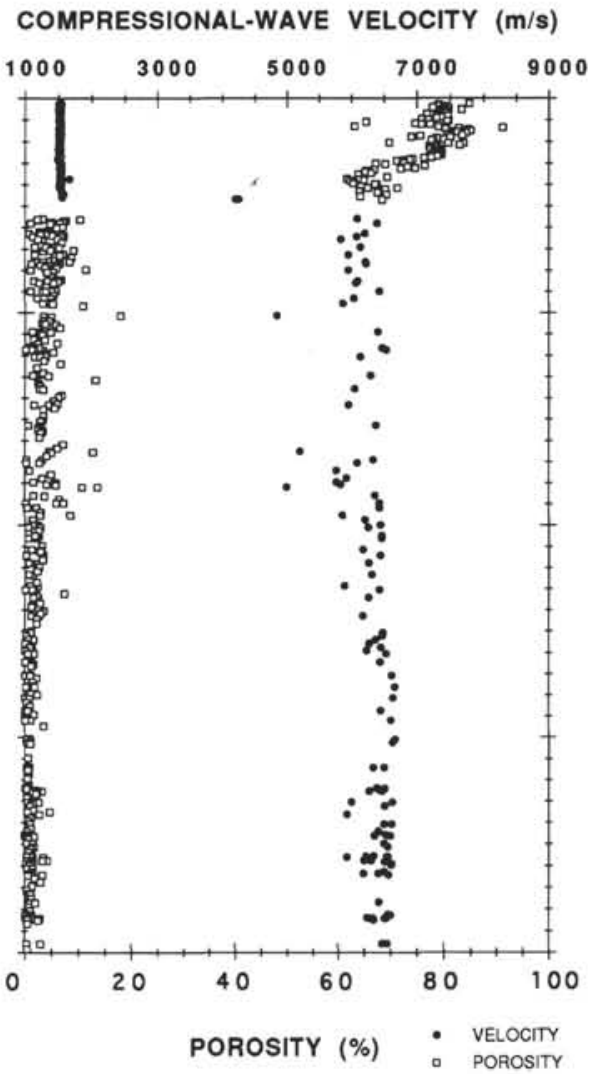

Figure 4. A. Discrete porosity and density measurements as a function of depth. B. Discrete porosity and velocity measurements as a function of depth. Velocities below the sediment cover are corrected to in-situ pressures.

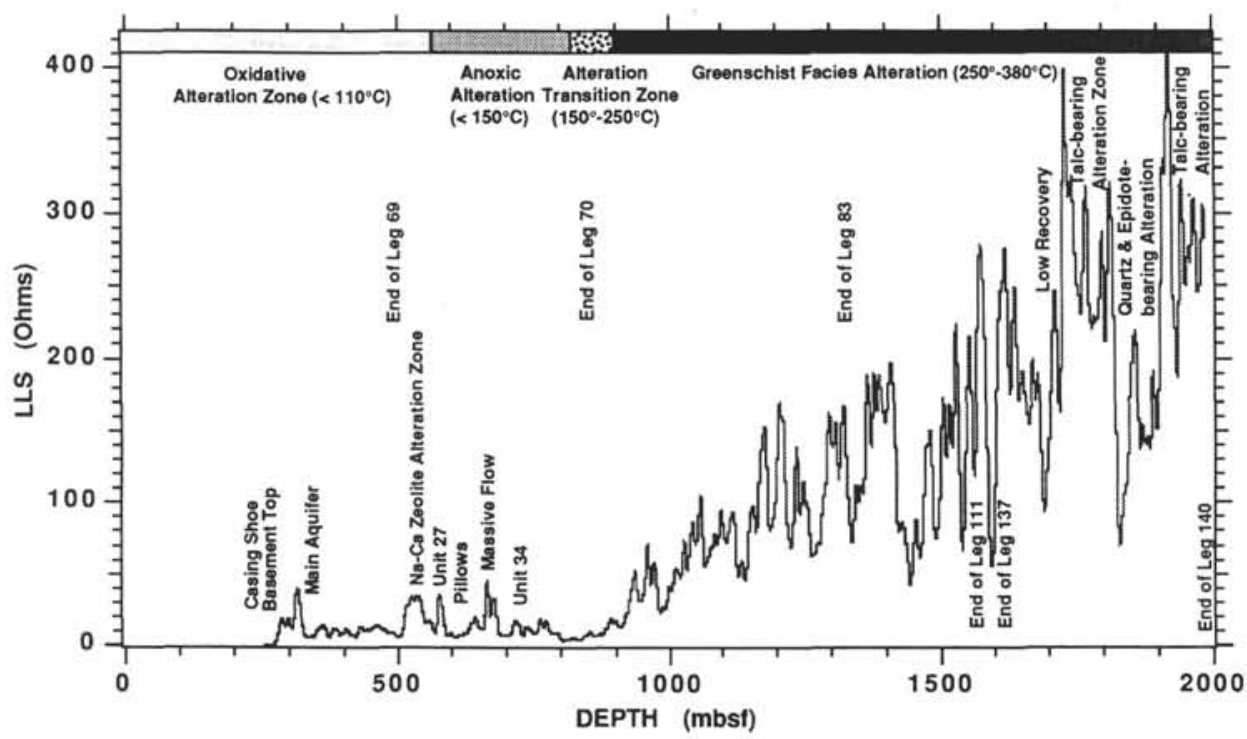

Figure 5. Shallow laterolog (LLS) as a function of depth obtained during Leg 140.

calcium, iron, and titanium. The samples from Legs 137 and 140 fall in a region between $m=21$ and $m=22$, and the slope seems to follow Birch's trend. Velocity measurements at $100 \mathrm{MPa}$ also were plotted as a function of bulk density and mean atomic weights (Fig. 6B). These particular velocity-pressure values were selected because they are closer to in-situ pressures, and the mean atomic weight lines were corrected for the pressure difference (Table 2.). The results also show most of the samples falling in a region between $\mathrm{m}=21$ and $\mathrm{m}=22$, but with a shift toward the $m=22$ line. The shift is considered to be a function of the cracks being partially open at this pressure and the fact that the velocity-pressure relationship begins to deviate from a linear relationship. Therefore, these results suggest that, at in-situ pressures, the velocities in Hole 504B are still controlled by mineralogical and porosity components. 
A

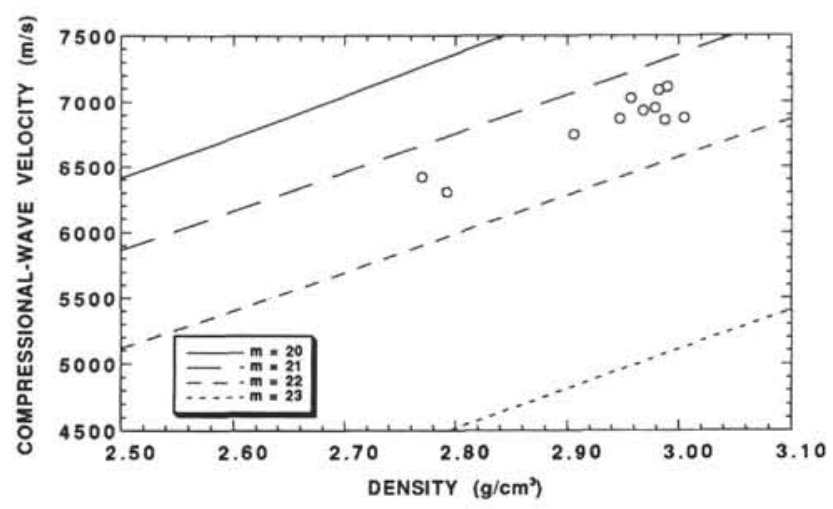

B

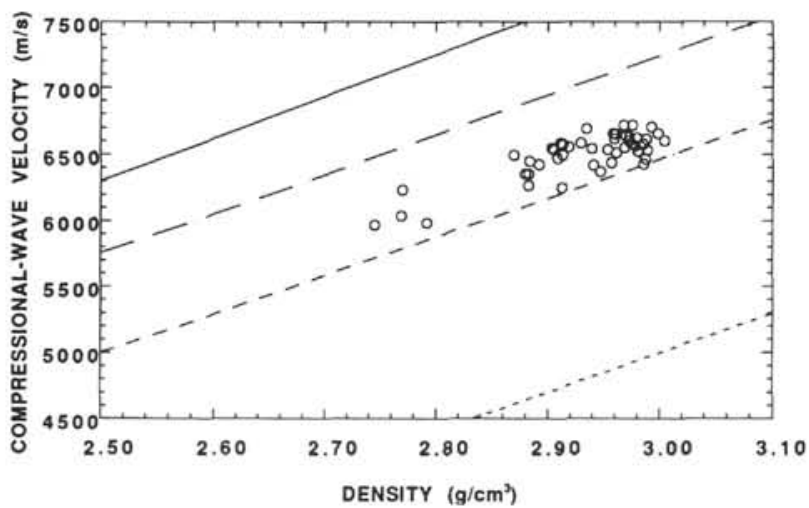

Figure 6. Velocity-density systematics for samples from Leg 140. Measurements were taken at (A) $600 \mathrm{MPa}$ to minimize the effect of crack porosity and (B) 100 $\mathrm{MPa}$ to look at the in-situ properties of the samples. Lines of constant mean atomic weights (m) are based on Birch's Law and corrected for pressure effects.

Table 2. Parameters used for calculating the lines of constant mean atomic weight $(\mathrm{MAW})$ at $600 \mathrm{MPa}$ and $100 \mathrm{MPa}$.

\begin{tabular}{crrrc}
\hline Average MAW & $\mathrm{a}_{1000}$ & $\mathrm{a}_{600}$ & $\mathrm{a}_{(60)}$ & $\mathrm{b}$ \\
\hline 20 & -1.423 & -1.513 & -1.623 & 3.169 \\
21 & -1.520 & -1.610 & -1.720 & 2.988 \\
22 & -2.123 & -2.213 & -2.323 & 2.929 \\
23 & -3.723 & -3.813 & -3.923 & 2.974
\end{tabular}

Note: These parameters were obtained from a least-squares solution of the form: $V_{p}=a+$ $\rho b$, where $V_{p}$ is in $\mathrm{km} / \mathrm{s}$ at $600 \mathrm{MPa}$ and $100 \mathrm{MPa}, \rho$ is in $\mathrm{g} / \mathrm{cm}^{3}$, a $a_{1000}$ is the velocity intercept for velocities at $1000 \mathrm{MPa}$ (Birch. 1960) in $\mathrm{km} / \mathrm{s}$, a intercept for velocities at $600 \mathrm{MPa}$ in $\mathrm{km} / \mathrm{s}$, a $\mathrm{a}_{\mathrm{f}}$ is the corrected intercept for velocities at $100 \mathrm{MPa}$ in $\mathrm{km} / \mathrm{s}$, and $\mathrm{b}$ is the slope of the lines in $(\mathrm{km} / \mathrm{s}) /\left(\mathrm{g} / \mathrm{cm}^{3}\right)$.
A similar analysis using previously published data at $600 \mathrm{MPa}$ (Wilkens et al., 1983; Christensen and Salisbury, 1985; Christensen et al., 1989) and the newly acquired values shows a similar trend with the highest velocities and densities recorded since Leg 111 (Fig. 7). This plot also shows that the samples recovered from Leg 69 through Leg 83 tend to deviate toward the $\mathrm{m}=22$ line, whereas the samples recovered since Leg 111 seem to remain closer to the $m=21$ boundary. These observations may imply that the differences correspond to zones characterized by a transition between oxic and anoxic types of alteration. These alteration conditions are significantly different from the conditions at the bottom of the hole, and perhaps a detailed study of the chemical compositions of the samples used for velocity measurements will reveal the specific chemical variations responsible for the observed deviations.

Previously published density-porosity values (Karato, 1983; Karato et al., 1983; Wilkens et al., 1983; Christensen and Salisbury, 1985; Christensen et al., 1989; Becker, Foss, et al., 1992) and newly acquired data show decreasing density trends with increasing porosity values (Fig. 8A and $8 \mathrm{~B}$ ). The slopes of the trends defined by the samples obtained during Legs 69,70 , and 83 , representing the pillow lavas as well as the upper section of the sheeted dikes, are shallower than the trend displayed by the samples from Legs 111, 137, and 140. These variations in slopes are thought to be related to the decrease in porosity due to the increasing overburden pressure and perhaps because most of the cracks are filled with alteration products toward the base of the hole, whereas in the upper section open cracks and vesicles are still present (Cann, Langseth, Honnorez, Von Herzen, White, et al., 1983). Statistical analyses of the data used for the diagrams are presented in Table 3.

\section{Velocity and Elastic Constants}

Velocity measurements corrected for in-situ pressures as a function of depth and plotted vs. porosity values show an increase in $V_{p}$ toward the bottom of Hole 504B (Fig. 4B). These results are quite different from the atmospheric pressure velocity values obtained as part of the shipboard analyses, which showed a marked decrease in compressional-wave velocities (Fig. 9). For the upper section of the hole, the atmospheric pressure values show an increase in velocity with depth, and as expected, they are lower than in-situ velocities. At an approximate depth of $1400 \mathrm{mbsf}$, the velocities recorded as part of the shipboard analyses begin to show a decreasing trend and a considerable difference from the in-situ values. This decreasing trend is attributed to the release of in-situ stresses that generate a series of microfractures that affect the velocity measurements at relatively low pressures. Therefore, these measurements point out the importance of measuring velocities as a function of confining pressure and the effects of unloading such stresses as the samples are brought to the surface from deep crustal sections.

Figure 4 also shows two zones at 1650 mbsf and between 1800 and $1850 \mathrm{mbsf}$ where low velocities correlate with low densities and higher porosity values. These values correlate with significant decreases in the resistivity $\log$ (LLS) that are found between $1600 \mathrm{mbsf}$ and $1700 \mathrm{mbsf}$ as well as between $1800 \mathrm{mbsf}$ and $1900 \mathrm{mbsf}$ (Fig. 5). Because the LLS is sensitive to both horizontal and vertical fracturing and measures pore fluid conduction (Pezard and Anderson, 1989), these results tend to suggest the presence of intervals that were or might still be hydrothermally active. These zones are characterized by high albite, talc, and mixed-layer clay contents (Dick, Erzinger, Stokking, et al., 1992), which tend to support the previous statement. The wavelength of a $30-\mathrm{Hz}$ source assuming $6.50 \mathrm{~km} / \mathrm{s}$ (Table 3 ) is $217 \mathrm{~m}$. From Figure 5, these low resistivity zones are approximately $100 \mathrm{~m}$ thick, which may suggest that because the velocities in these zones should be lower than the average for the entire sheeted dikes section, the previously reported $\mathrm{E}_{5}$ reflector (Becker, Sakai, et al., 1988; Collins et al., 1989) could be a product of a low velocity zone. 


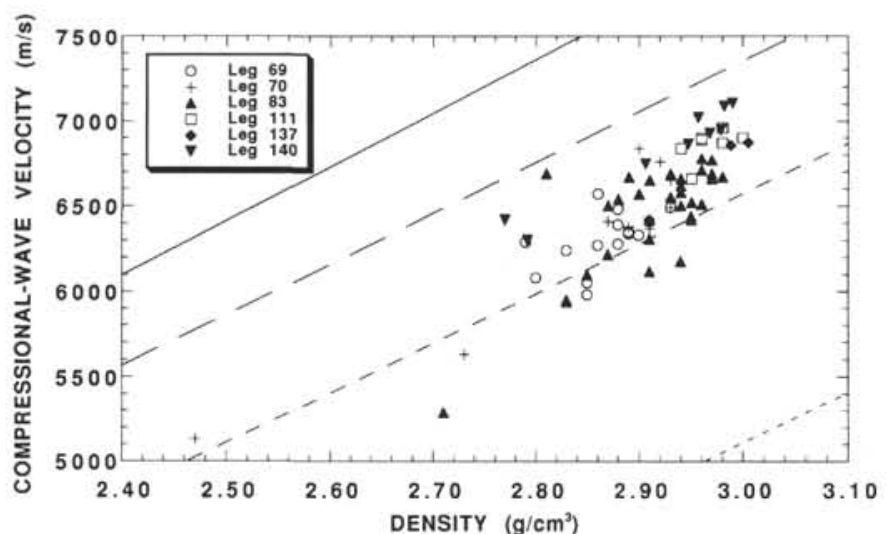

Figure 7. Velocity-density systematics for Hole 504B used to compare the results obtained through the entire drill-core section. Lines of constant mean atomic weights $(\mathrm{m})$ are based on Birch's Law and corrected to $600-\mathrm{MPa}$ pressure. Values same as in Figure 6A.

\section{A}

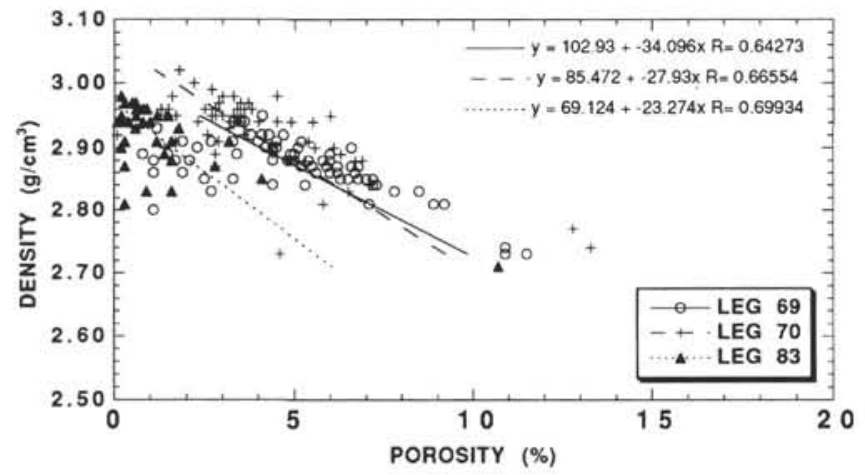

\section{B}

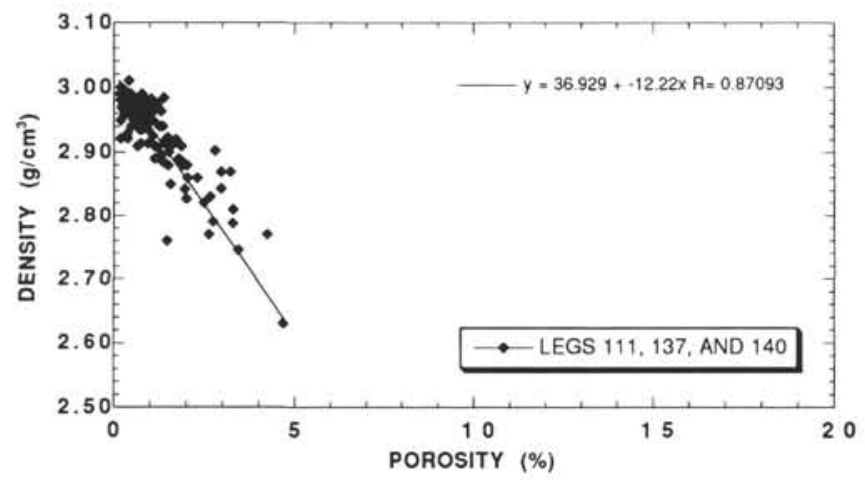

Figure 8. Porosity-density relationships for the rocks recovered during (A) Legs 69, 70, and 83, and (B) Legs 111, 137, and 140.

The $V_{p} / V_{s}$ ratio is a parameter widely used by seismologists for interpreting seismic data, and Poisson's ratio is a measure of the lateral contraction with respect to the longitudinal extension. A $V_{p}-V_{s}$ plot at $100 \mathrm{MPa}$ (Fig. 10A) shows that most of the samples from Hole 504B fall in a region bounded by lines of constant Poisson's ratio that range from 0.25 to 0.30 . Several samples from the pillow lavas and upper

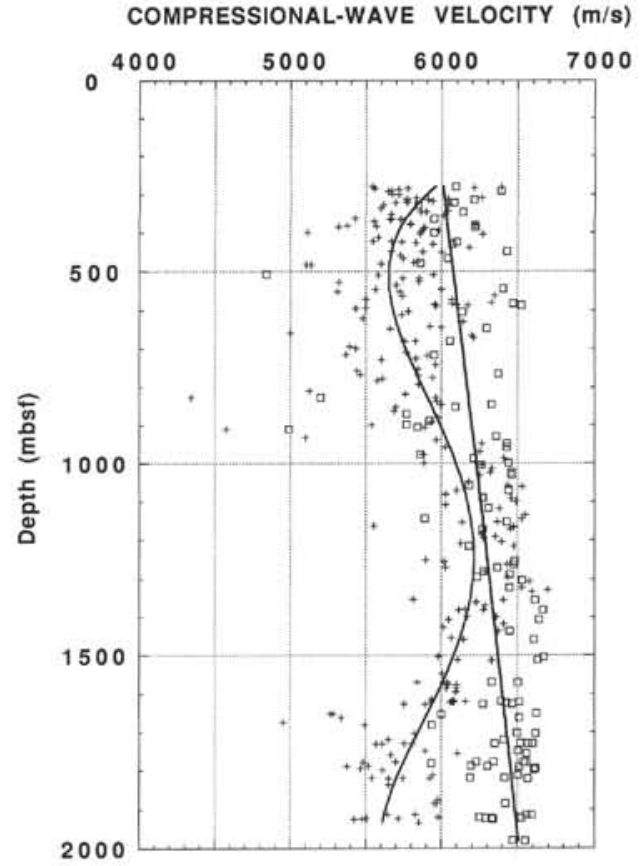

Figure 9. Compressional-wave velocity values from Hole 504B measured at atmospheric (pluses) and in-situ pressures (squares). Polynomial fits of $2^{\text {nd }}$ $\left(V_{p}\right.$ in-situ) and $4^{\text {th }}$ orders ( $V_{p}$ atmospheric) were applied to the data to show velocity trends.

sheeted dikes section (Legs 69, 70, and 83) fall above the 0.30 line, whereas the samples from the middle to lower sheeted dikes section (Legs 111, 137, and 140) fall below the 0.30 line. A comparison of laboratory velocity measurements from Hole 504B recorded at in-situ pressures with refraction data from the northeastern Pacific (Spudich and Orcutt, 1980; Au and Clowes, 1984) shows that most of the Hole 504B data fall along the same trend with a similar range of values (Fig. 10B). In the past, seismic refraction experiments of the upper oceanic crust (Spudich and Orcutt. 1980; Au and Clowes, 1984) have shown anomalously low values for Poisson's ratio $(0.20-0.25)$ at depths ranging from $1.0 \mathrm{~km}$ to approximately $2.0 \mathrm{~km}$ below seafloor (Fig. 10C). The results from Hole 504B laboratory samples display a similar trend albeit with somewhat higher values for Poisson's ratio (Figs. 10C and 11A). In Hole 504B, a decrease in both Poisson and $V_{p} / V_{s}$ ratios is observed as these parameters are plotted as functions of depth and corrected for in-situ pressures (Fig. 11A).

Shear-wave velocities are extremely sensitive to fractures, having a tendency to be slower propagating perpendicular to cracks and becoming faster as fractures close with increasing pressure. The decrease in $V_{p} / V_{s}$ and Poisson ratios may suggest the presence of microfractures causing a larger increase in $V_{s}$ than in $V_{p}$ with depth. Logging data (Pezard and Anderson, 1989; Dick, Erzinger, Stokking, et al., 1992) suggest that high fracture porosity recorded in the first few hundred meters of Hole 504B changes at $406 \mathrm{mbsf}$ from a zone of both horizontal and vertical networks to a regime of lower fracture porosity mainly composed of vertical fractures. However, laboratory measurements performed on Leg 137/140 samples show both Vs1 (propagating and vibrating horizontally) and Vs2 (propagating horizontally and vibrating vertically) to be relatively isotropic (Table 1), suggesting no preferred fracture orientations on a sample scale. The lack of shear-wave anisotropy and the absence of a significant mineral fabric (Dick, Erzinger, Stokking, et al., 1992) also indicate that the changes in Poisson's ratio are not due to a preferred mineral orientation. Therefore, the changes in elastic properties with depth must be explained in terms of microcrack density and aspect ratios within relatively isotropic samples. 

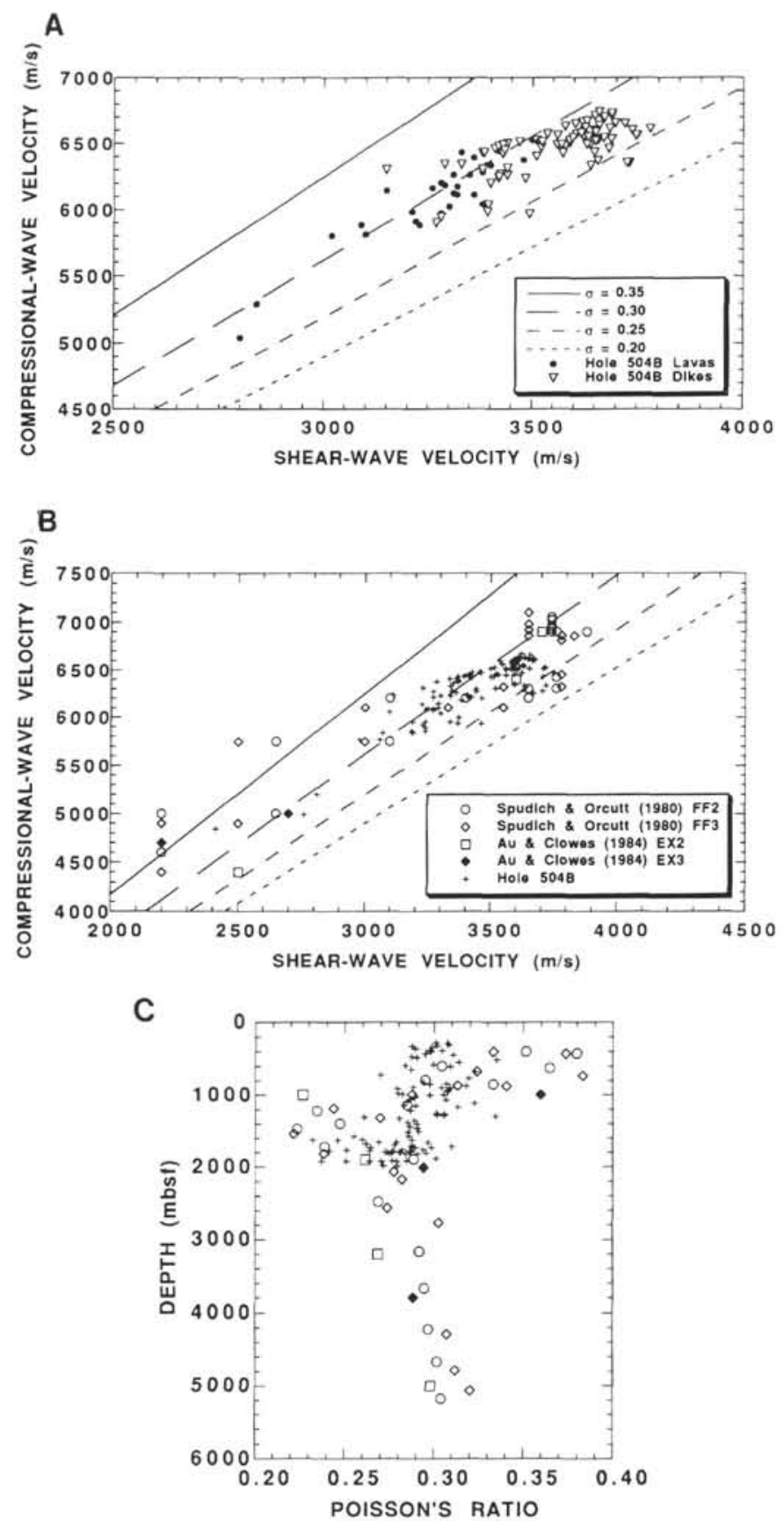

Figure 10. A. Compressional- and shear-wave velocities at $100 \mathrm{MPa}$ for the rock drilled at Hole 504B. Black dots represent the pillow lavas, triangles symbolize the sheeted dikes, and lines represent constant Poisson's ratio. B. Comparison of Hole 504B velocity data with seismic refraction lines from the northeastern Pacific. Laboratory measurements are plotted at in-situ pressures. C. Poisson's ratio as a function of depth and in-situ pressure for Hole 504B and the northeastern Pacific data. Symbols are the same as in Figure 10B.

Theories of cracked media (Walsh, 1969; Kuster and Toksöz, 1974; O'Connell and Budianski, 1974; Garbin and Knopoff, 1975; Watt, et al., 1976; Hudson, 1980) used for isotropic thin-cracked models have previously shown a decrease in Poisson's ratio with decreasing porosity (Shearer, 1988). For thin-cracked models (aspect ratio $=0.001)$ a decrease in Poisson's ratio with decreasing porosity reflects a proportionally higher increase in S-wave velocities than in
P-waves (Shearer, 1988). These results seem to compare favorably with the lower porosity values, lower Poisson ratios, and relatively isotropic conditions observed within the bottom of Hole 504B.

The results from Hole 504B laboratory samples have shown that in the upper oceanic crust between 1 and $2 \mathrm{~km}$ below seafloor the Poisson's ratio values match the range of values obtained from refraction experiments, although, on the average, the Hole 504B samples display higher values (Figs. 10C and 11A). Theoretical isotropic thick-cracked models (Hyndman, 1979; Shearer, 1988) have shown a decrease in Poisson's ratio with an increase in porosity values because the velocity reduction in P-waves is more important. Therefore, one possible explanation for the difference in magnitude between both data sets can be attributed to crack aspect ratios and the scales of observations. The presence of large-scale low resistivity zones (Fig. 5) suggests the possibility of thick fractures within the sheeted dikes section, which can decrease values of Poisson's ratio obtained through seismic refraction experiments. Microfractures within laboratory samples from Hole 504B also reduce Poisson's ratio but the effect may not be as significant.

Another possibility for the reduction of Poisson's ratio values obtained from refraction experiments (Spudich and Orcutt, 1980; Au and Clowes, 1984) may be attributed to pore pressure effects. Laboratory studies in basalts have shown that increasing pore pressure at constant confining pressure reduces shear-wave velocities significantly more than compressional-wave velocities, causing Poisson's ratio to increase (Christensen, 1984). These changes are substantially greater for basalts than for dolerites presumably because of the higher porosity in basalts and complicated by the presence of vesicles and microfractures (Christensen, 1984). If pore pressure is equivalent to hydrostatic pressure in the upper $2 \mathrm{~km}$ of the oceanic crust, Poisson's ratio will decrease with increasing depth (Christensen, 1984). Critical to this will be the percentage of interconnected pore spaces and the pore geometry, including the aspect ratios of fractures. However, a zone within the upper crustal section drilled at Hole 504B has fluctuated back and forth from underpressured to approaching hydrostatic equilibrium through time perhaps due to movement along a fault. Downhole temperature measurements throughout the history of Hole 504B have shown significant fluctuations with time in the upper basement where a distinctive underpressured zone above $400 \mathrm{mbsf}$ (Anderson and Zoback, 1982; Becker et al., 1983a; 1983b; 1985; Becker, Foss, et al., 1992) gradually approaches hydrostatic pressure (Gable et al., 1989; Dick, Erzinger, Stokking, et al., 1992), whereas below 400 mbsf, the basement temperatures and porosity values suggest a relatively impermeable crust. These apparent variations along with the decrease in porosity and permeability with depth (Becker, 1989; Dick, Erzinger, Stokking, et al., 1992) suggest that the effects of pore pressure in upper oceanic crust are more significant in the pillow lavas than in the lower sheeted dikes section.

Finally, variations in the mineralogical composition of the upper oceanic crust also can contribute to changes in elastic properties. During metamorphic processes the crystallization of quartz can account for the reduction of Poisson's ratio. Ophiolite studies in Troodos (Baragar et al., 1989; Robinson, 1989; Robinson et al., 1991) have reported the presence of interstitial quartz throughout the sheeted dikes section, and some low Poisson's ratio values $(\sigma \leq 0.25)$ have been recorded (Christensen and Salisbury, 1989). In oceanic rocks, compressional-wave velocities tend to decrease with an increase in $\mathrm{SiO}_{2}$ content (Iturrino and Christensen, 1991). However, throughout the sheeted dikes section, the rocks from Hole 504B have a considerably lower $\mathrm{SiO}_{2}$ content (Dick, Erzinger, Stokking, et al., 1992) than the rocks from Troodos (Baragar et al., 1989). Also, the $\mathrm{SiO}_{2}$ content of the basalts drilled in Hole 504B seems to be similar to the values obtained in the sheeted dikes section (Dick, Erzinger, Stokking, et al., 1992). Therefore, even if up to this point no detailed chemical analyses have been performed on the rocks used for velocity measurements, the low Poisson's ratio values measured at the bottom of Hole 504B seem to be related to the presence of fractures and their aspect ratios. 


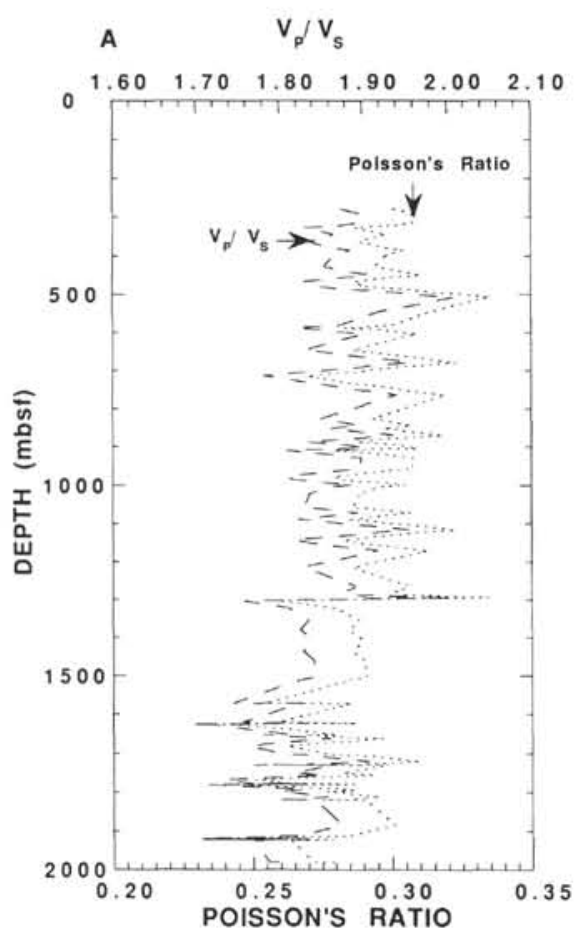

B BULK MODULUS (Mb)

$\begin{array}{llllllll}0.30 & 0.40 & 0.50 & 0.60 & 0.70 & 0.80 & 0.90\end{array}$

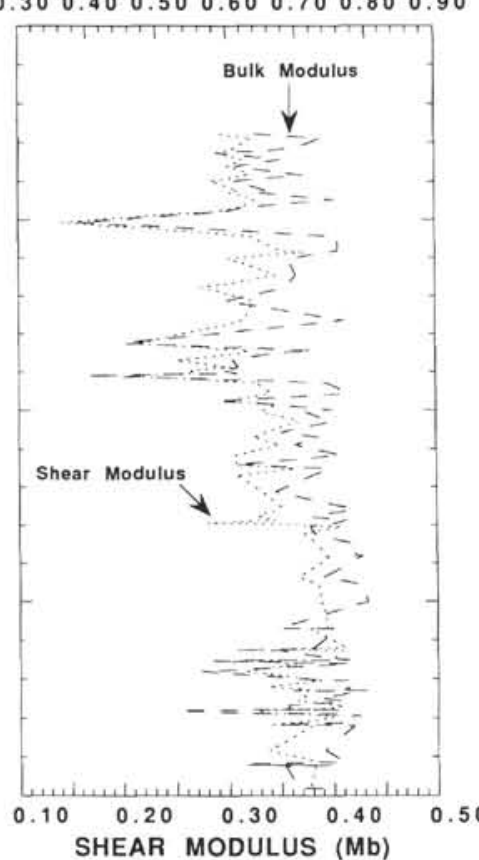

Figure 11. A plot of elastic constants calculated from discrete measurements of laboratory samples from Hole 504B and displayed as a function of depth and in-situ pressures. A. Poisson (dotted) and $V_{p} / V_{s}$ (dashed) ratios. B. Bulk (dashed) and shear (dotted) moduli. Dotted and dashed lines simply connect discrete measurements for illustration purposes and do not represent a continuous downhole measurement.
The bulk modulus is the stress-strain relationship under simple hydrostatic pressure, whereas the shear modulus or rigidity modulus is the stress-strain relationship for simple shear. In Hole 504B, both parameters show an increase with depth except for some variations observed in the last $300 \mathrm{mbsf}$ (Fig. 11B). These variations correlate with similar variations observed in the LLS and can be attributed to variations in fracture porosity. In theoretical models with small aspect ratios $(<0.1)$ the shear modulus decreases by a fixed amount determined only by the crack density, whereas the bulk modulus remains unchanged for thin cracks but increases with crack thickness (Shearer, 1988). Therefore, the relative increase with depth of both shear and bulk moduli displayed by laboratory measurements represents a set of samples with relatively low crack density and small aspect ratios. However, there are well-defined zones in the last $300 \mathrm{mbsf}$ where the crack density and aspect ratios seem to be higher.

$V_{p} / V_{s}$ ratios, Poisson ratios $(\sigma)$, bulk moduli $(\mathrm{K})$, and shear moduli $(\mu)$ calculated at selected pressures from measured densities and velocities are given in Table 4 . These values are important for drilling considerations because in a general way rock fracture strength is proportional to the elastic moduli. In all cases, preexisting fractures reduce the effective elastic stiffness and serve as sources of macroscopic failure by fracturing under the drill bit. These measurements can assist drilling engineers and scientists in making load-pressure decisions and bit selections and may also allow them to better estimate the life span of a core bit when drilling into fracture zones and deep crustal sections.

\section{Ophiolite Data}

Ophiolites are thought to be onshore exposures of ocean crust; therefore, physical properties from Hole 504B should correlate with results from previous ophiolite studies. Some of the best studied ophiolite exposures include the Bay of Islands massifs in Newfoundland and the Semail region in Oman.

The North Arm and Blow Me Down massifs are two of the four major ophiolite sections exposed in western Newfoundland. Based on petrology and geochemistry (Williams and Malpas, 1972; Malpas, 1977, 1978; Jacobsen and Wasserburg, 1979; Elthon, et al., 1982, 1984; Casey et al., 1985), the Bay of Islands ophiolites have been
Table 3. Statistical analyses for the physical properties data from Hole 504B.

\begin{tabular}{|c|c|c|}
\hline Statistical Analyses & Legs 69,70 , and 83 & Legs 111,137 , and 140 \\
\hline \multicolumn{3}{|l|}{ Density $\left(\mathrm{g} / \mathrm{cm}^{3}\right)$} \\
\hline Number of samples & 171 & 114 \\
\hline Mean & 2.90 & 2.93 \\
\hline Maximum value & 3.02 & 3.01 \\
\hline Minimum value & 2.71 & 2.63 \\
\hline Median & 2.90 & 2.94 \\
\hline Standard deviation & 0.06 & 0.06 \\
\hline \multicolumn{3}{|l|}{ Porosity (\%) } \\
\hline Number of samples & 171 & 115 \\
\hline Mean & 3.86 & 1.16 \\
\hline Maximum value & 13.30 & 4.68 \\
\hline Minimum value & 0.12 & 0.17 \\
\hline Median & 3.50 & 0.94 \\
\hline Standard deviation & 2.62 & 0.91 \\
\hline \multicolumn{3}{|l|}{$\mathrm{V}_{\mathrm{p}}(\mathrm{m} / \mathrm{s})$} \\
\hline Number of samples & 57 & 55 \\
\hline Mean & 6237 & 6506 \\
\hline Maximum value & 6610 & 6740 \\
\hline Minimum value & 5040 & 5967 \\
\hline Median & 6300 & 6542 \\
\hline Standard deviation & 300.4 & 176.4 \\
\hline \multicolumn{3}{|l|}{$\mathrm{V}_{\mathrm{s}}(\mathrm{m} / \mathrm{s})$} \\
\hline Number of samples & 57 & 54 \\
\hline Mean & 3354 & 3618 \\
\hline Maximum value & 3750 & 3820 \\
\hline Minimum value & 2800 & 3385 \\
\hline Median & 3380 & 3640 \\
\hline Standard deviation & 170.9 & 99.7 \\
\hline
\end{tabular}

described as a mid-ocean-ridge complex in origin. The North Arm massif represents the best section for comparison with Hole 504B because it has a well-defined sediment-basalt contact and there is good stratigraphic control throughout the upper crust (Christensen and Salisbury, 1982), whereas in the Blow Me Down massif, the upper basalt section is stripped by erosion and the existent section is characterized by pumpellyite-prehnite alteration (Salisbury and Christensen, 1978). Therefore, laboratory measurements from the Bay of Islands ophiolites were used for a direct comparison because the upper sec- 
Table 4. Elastic constants calculated from laboratory measured velocities and densities for the rocks recovered during Legs 137 and 140.

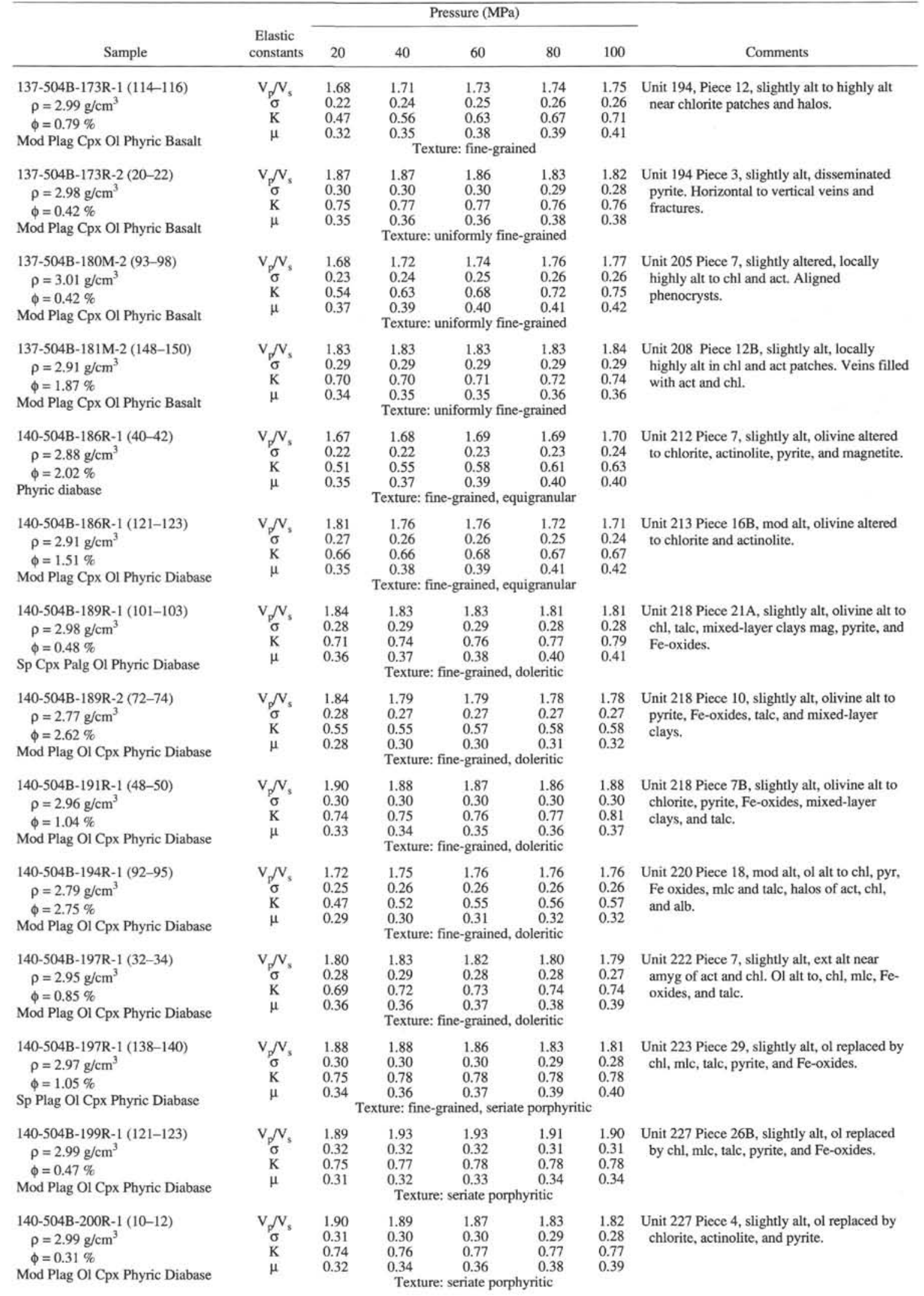


Table 4 (continued).

\begin{tabular}{|c|c|c|c|c|c|c|c|}
\hline \multirow[b]{2}{*}{ Sample } & \multirow[b]{2}{*}{$\begin{array}{l}\text { Elastic } \\
\text { constants }\end{array}$} & \multicolumn{5}{|c|}{ Pressure (MPa) } & \multirow[b]{2}{*}{ Comments } \\
\hline & & 20 & 40 & 60 & 80 & 100 & \\
\hline $\begin{array}{l}140-504 \mathrm{~B}-200 \mathrm{R}-2(41-43) \\
\quad \begin{array}{l}\rho \\
=2.98 \mathrm{~g} / \mathrm{cm}^{3}\end{array} \\
\phi=0.91 \% \\
\text { Mod Plag OI Cpx Phyric Diabase }\end{array}$ & $\begin{array}{l}\mathrm{V}_{\mathrm{p}} / \mathrm{V}_{\mathrm{s}} \\
\mathrm{K} \\
\mu\end{array}$ & $\begin{array}{l}1.93 \\
0.31 \\
0.77 \\
0.33\end{array}$ & $\begin{array}{c}1.88 \\
0.30 \\
0.77 \\
0.35 \\
\text { Text }\end{array}$ & $\begin{array}{c}1.87 \\
0.30 \\
0.79 \\
0.36 \\
\text { eriate p }\end{array}$ & $\begin{array}{l}1.84 \\
0.29 \\
0.78 \\
0.38 \\
\text { ritic }\end{array}$ & $\begin{array}{l}1.83 \\
0.29 \\
0.78 \\
0.39\end{array}$ & $\begin{array}{l}\text { Unit } 227 \text { Piece } 6 \text {, slightly alt, ol replaced by } \\
\text { chl, act, and pyrite with trace amounts of Fe- } \\
\text { oxides. }\end{array}$ \\
\hline $\begin{array}{l}140-504 \mathrm{~B}-200 \mathrm{R}-2(44-46) \\
\quad \begin{array}{l}\rho \\
=2.99 \mathrm{~g} / \mathrm{cm}^{3}\end{array} \\
\phi=0.38 \% \\
\text { Mod Plag OI Cpx Phyric Diabase }\end{array}$ & $\begin{array}{l}\mathrm{V}_{\mathrm{p}} / \mathrm{V}_{\mathrm{s}} \\
\mathrm{K} \\
\mu\end{array}$ & $\begin{array}{l}1.68 \\
0.23 \\
0.42 \\
0.28\end{array}$ & $\begin{array}{c}1.72 \\
0.24 \\
0.54 \\
0.33 \\
\text { Text }\end{array}$ & $\begin{array}{c}1.75 \\
0.26 \\
0.63 \\
0.36 \\
\text { eriate p }\end{array}$ & $\begin{array}{l}1.77 \\
0.26 \\
0.69 \\
0.39 \\
\text { ritic }\end{array}$ & $\begin{array}{l}1.78 \\
0.27 \\
0.74 \\
0.40\end{array}$ & $\begin{array}{l}\text { Unit } 227 \text { Piece } 6 \text {, slightly alt, ol replaced by } \\
\text { chl, act, and pyrite with trace amounts of Fe- } \\
\text { oxides. }\end{array}$ \\
\hline $\begin{array}{l}\text { 140-504B-200R-2 (73-75) } \\
\qquad \begin{array}{l}\rho=2.97 \mathrm{~g} / \mathrm{cm}^{3} \\
\phi=0.34 \%\end{array} \\
\text { Mod Plag Ol Cpx Phyric Diabase }\end{array}$ & $\begin{array}{l}\mathrm{V}_{\mathrm{p}} / \mathrm{V}_{\mathrm{s}} \\
\sigma \\
\mathrm{K} \\
\mu\end{array}$ & $\begin{array}{l}1.92 \\
0.31 \\
0.77 \\
0.33\end{array}$ & $\begin{array}{c}1.91 \\
0.31 \\
0.79 \\
0.34 \\
\text { Text }\end{array}$ & $\begin{array}{c}1.90 \\
0.31 \\
0.79 \\
0.35 \\
\text { eriate p }\end{array}$ & $\begin{array}{r}1.88 \\
0.30 \\
0.80 \\
0.37 \\
\text { ritic }\end{array}$ & $\begin{array}{l}1.86 \\
0.29 \\
0.80 \\
0.38\end{array}$ & $\begin{array}{l}\text { Unit } 227 \text { Piece } 9 \text {, slightly alt, ol replaced by } \\
\text { chl, act, and pyrite with trace amounts of Fe- } \\
\text { oxides. }\end{array}$ \\
\hline $\begin{array}{l}\text { 140-504B-203R-1 (57-59) } \\
\qquad \begin{array}{l}\rho=2.90 \mathrm{~g} / \mathrm{cm}^{3} \\
\phi=1.53 \%\end{array} \\
\text { Mod Plag Cpx OI Phyric Diabase }\end{array}$ & $\begin{array}{l}\mathrm{V}_{\mathrm{p}} / \mathrm{V}_{5} \\
\sigma \\
\mathrm{K} \\
\mu\end{array}$ & $\begin{array}{c}1.83 \\
0.29 \\
0.71 \\
0.35 \\
\text { Text }\end{array}$ & $\begin{array}{c}1.83 \\
0.29 \\
0.72 \\
0.36 \\
\text { microc }\end{array}$ & $\begin{array}{c}1.83 \\
0.29 \\
0.73 \\
0.36 \\
\text { line to } \mathrm{f}\end{array}$ & $\begin{array}{c}1.82 \\
0.28 \\
0.73 \\
0.37 \\
\text { rained }\end{array}$ & $\begin{array}{c}1.82 \\
0.28 \\
0.74 \\
0.38 \\
\text { nitic }\end{array}$ & $\begin{array}{l}\text { Unit } 232 \text { Piece } 15 \text {, highly alt to act, chl, alb, } \\
\text { and titanite. Mats of act rimmed by chl. } \\
\text { Pods of chl. }\end{array}$ \\
\hline $\begin{array}{l}140-504 \mathrm{~B}-205 \mathrm{R}-1(3-5) \\
\quad \rho=2.91 \mathrm{~g} / \mathrm{cm}^{3} \\
\quad \phi=1.58 \% \\
\text { Sp Plag Cpx Ol Phyric Diabase }\end{array}$ & $\begin{array}{l}\mathrm{V}_{\mathrm{p}} / \mathrm{V}_{\mathrm{s}} \\
\mathrm{K} \\
\mu\end{array}$ & $\begin{array}{c}1.85 \\
0.29 \\
0.74 \\
0.35 \\
\text { Text }\end{array}$ & $\begin{array}{r}1.85 \\
0.29 \\
0.75 \\
0.36 \\
\text { microc }\end{array}$ & $\begin{array}{c}1.86 \\
0.30 \\
0.76 \\
0.36 \\
\text { line to fi }\end{array}$ & $\begin{array}{c}1.85 \\
0.29 \\
0.77 \\
0.37 \\
\text { rained }\end{array}$ & $\begin{array}{r}1.85 \\
0.29 \\
0.77 \\
0.37 \\
\text { anitic }\end{array}$ & $\begin{array}{l}\text { Unit } 232 \text { Piece } 1 \text {, highly alt to act, alb, chl, } \\
\text { and titanite. Rare ol replaced by act, chl, } \\
\text { talc, and pyrite. }\end{array}$ \\
\hline $\begin{array}{l}\text { 140-504B-208R-1 (4-7) } \\
\quad \rho=2.98 \mathrm{~g} / \mathrm{cm}^{3} \\
\quad \phi=0.54 \% \\
\text { Mod Cpx OI Plag Phyric Diabase }\end{array}$ & $\begin{array}{l}\mathrm{V}_{\mathrm{p}} / \mathrm{V}_{\mathrm{s}} \\
\mathrm{K} \\
\mu\end{array}$ & $\begin{array}{l}1.63 \\
0.20 \\
0.40 \\
0.30\end{array}$ & $\begin{array}{l}1.70 \\
0.23 \\
0.53 \\
0.34 \\
\text { Textu }\end{array}$ & $\begin{array}{c}1.74 \\
0.25 \\
0.62 \\
0.37 \\
\text { e-grain }\end{array}$ & $\begin{array}{l}1.77 \\
0.26 \\
0.69 \\
0.38 \\
\text { leritic }\end{array}$ & $\begin{array}{l}1.78 \\
0.27 \\
0.74 \\
0.40\end{array}$ & $\begin{array}{l}\text { Unit } 237 \text { Piece 1, groundmass alt to act, chl, } \\
\text { alb, and titanite. Ol alt to chl, talc, mic, } \\
\text { pyrite, and Fe-oxide. }\end{array}$ \\
\hline $\begin{array}{l}\text { 140-504B-208R-1 (85-87) } \\
\quad \begin{array}{l}\rho=2.88 \mathrm{~g} / \mathrm{cm}^{3} \\
\phi=1.51 \%\end{array} \\
\text { Mod Ol Plag Cpx Phyric Diabase }\end{array}$ & $\begin{array}{l}\mathrm{V}_{p} / \mathrm{V}_{\mathrm{s}} \\
\mathrm{K} \\
\mu\end{array}$ & $\begin{array}{r}1.82 \\
0.28 \\
0.64 \\
0.32 \\
\mathrm{~T}\end{array}$ & $\begin{array}{c}1.82 \\
0.28 \\
0.65 \\
0.33 \\
\text { ire: fine }\end{array}$ & $\begin{array}{c}1.83 \\
0.29 \\
0.66 \\
0.33 \\
\text { ed equi } \\
\text { crystall }\end{array}$ & $\begin{array}{c}1.83 \\
0.29 \\
0.67 \\
0.33 \\
\text { lar dole }\end{array}$ & $\begin{array}{l}1.83 \\
0.29 \\
0.68 \\
0.34 \\
c,\end{array}$ & $\begin{array}{l}\text { Unit } 239 \text { Piece } 19 \mathrm{~A} \text {, slightly alt to act, chl, } \\
\text { alb, and titanite. Ol alt to chl, talc, mlc, } \\
\text { pyrite, and Fe-oxides. }\end{array}$ \\
\hline $\begin{array}{l}\text { 140-504B-208R-1 (132-134) } \\
\quad \rho=2.97 \mathrm{~g} / \mathrm{cm}^{3} \\
\phi=1.12 \% \\
\text { Mod O1 Plag Cpx Phyric Diabase }\end{array}$ & $\begin{array}{l}\mathrm{V}_{p} / \mathrm{V}_{\mathrm{s}} \\
\mathrm{K}_{\mathrm{K}} \\
\mu\end{array}$ & $\begin{array}{r}1.88 \\
0.31 \\
0.71 \\
0.32 \\
\mathrm{~T}\end{array}$ & $\begin{array}{c}1.89 \\
0.30 \\
0.74 \\
0.34 \\
\text { re: fine }\end{array}$ & $\begin{array}{c}1.87 \\
0.30 \\
0.76 \\
0.36 \\
\text { ed equi } \\
\text { cerystall }\end{array}$ & $\begin{array}{c}1.84 \\
0.29 \\
0.77 \\
0.38 \\
\text { lar dol }\end{array}$ & $\begin{array}{l}1.82 \\
0.28 \\
0.77 \\
0.40 \\
c,\end{array}$ & $\begin{array}{l}\text { Unit } 239 \text { Piece } 25 \mathrm{~B} \text {, slightly alt to act, chl, } \\
\text { alb, and titanite. Ol alt to chl, talc, mlc, } \\
\text { pyrite, and Fe-oxides. }\end{array}$ \\
\hline $\begin{array}{l}140-504 \mathrm{~B}-208 \mathrm{R}-2(10-12) \\
\quad \rho=2.99 \mathrm{~g} / \mathrm{cm}^{3} \\
\phi=0.38 \% \\
\text { Mod Ol Plag Cpx Phyric Diabase }\end{array}$ & $\begin{array}{l}\mathrm{V}_{p} / \mathrm{V}_{\mathrm{s}} \\
\sigma \\
\mathrm{K} \\
\mu\end{array}$ & $\begin{array}{r}1.82 \\
0.28 \\
0.69 \\
0.35 \\
\mathrm{~T}\end{array}$ & $\begin{array}{c}1.81 \\
0.28 \\
0.73 \\
0.37 \\
\text { Ire: fine }\end{array}$ & $\begin{array}{c}1.81 \\
0.28 \\
0.74 \\
0.38 \\
\text { ed equi } \\
\text { cerystall }\end{array}$ & $\begin{array}{c}1.79 \\
0.28 \\
0.75 \\
0.39 \\
\text { lar dole }\end{array}$ & $\begin{array}{l}1.79 \\
0.28 \\
0.77 \\
0.40 \\
c,\end{array}$ & $\begin{array}{l}\text { Unit } 239 \text { Piece } 2 \text {, slightly alt with local } \\
\text { intense alt patches, alt to act, chl, alb, } \\
\text { titanite, and tr of epidote. }\end{array}$ \\
\hline $\begin{array}{l}140-504 \mathrm{~B}-208 \mathrm{R}-3(6-8) \\
\quad \rho=2.75 \mathrm{~g} / \mathrm{cm}^{3} \\
\phi=3.44 \% \\
\text { Mod Ol Plag Cpx Phyric Diabase }\end{array}$ & $\begin{array}{l}\mathrm{V}_{\mathrm{p}} / \mathrm{V}_{\mathrm{s}} \\
\mathrm{K}_{\mathrm{K}} \\
\mu\end{array}$ & $\begin{array}{r}1.71 \\
0.24 \\
0.49 \\
0.31 \\
\mathrm{~T}\end{array}$ & $\begin{array}{c}1.71 \\
0.25 \\
0.51 \\
0.31 \\
\text { ure: fine }\end{array}$ & $\begin{array}{c}1.73 \\
0.25 \\
0.53 \\
0.32 \\
\text { ed equi } \\
\text { cerystall }\end{array}$ & $\begin{array}{c}1.73 \\
0.24 \\
0.53 \\
0.33 \\
\text { lar dole }\end{array}$ & $\begin{array}{l}1.71 \\
0.24 \\
0.53 \\
0.33 \\
c,\end{array}$ & $\begin{array}{l}\text { Unit } 239 \text { Piece } 1 \text {, mod alt with intense alt } \\
\text { near chl, act, and alb halos and rimmed act } \\
\text { amygdules. }\end{array}$ \\
\hline $\begin{array}{l}\text { 140-504B-209R-1 (103-105) } \\
\quad \rho=2.77 \mathrm{~g} / \mathrm{cm}^{3} \\
\quad \begin{array}{l}\mathrm{c}^{3} \\
=4.23 \%\end{array} \\
\text { Mod Ol Plag Cpx Phyric Diabase }\end{array}$ & $\begin{array}{l}\mathrm{V}_{\mathrm{p}} / \mathrm{V}_{\mathrm{s}} \\
\mathrm{K} \\
\mu\end{array}$ & $\begin{array}{l}1.73 \\
0.25 \\
0.51 \\
0.31\end{array}$ & $\begin{array}{r}1.76 \\
0.26 \\
0.57 \\
0.32 \\
\text { xture: }\end{array}$ & $\begin{array}{l}1.78 \\
0.27 \\
0.60 \\
0.33\end{array}$ & $\begin{array}{c}1.79 \\
0.27 \\
0.62 \\
0.33 \\
\text { dolerit }\end{array}$ & $\begin{array}{l}1.79 \\
0.27 \\
0.63 \\
0.34\end{array}$ & $\begin{array}{l}\text { Unit } 240 \text { Piece 14B, slightly alt; highly alt } \\
\text { near halos to act, chl, alb, and titanite with } \\
\text { minor epidote. }\end{array}$ \\
\hline $\begin{array}{l}\text { 140-504B-209R-2 (5-7) } \\
\qquad \begin{array}{l}\rho=2.89 \mathrm{~g} / \mathrm{cm}^{3} \\
\phi=1.26 \%\end{array} \\
\text { Mod Ol Plag Cpx Phyric Diabase }\end{array}$ & $\begin{array}{l}\mathrm{V}_{\mathrm{p}} / \mathrm{V}_{\mathrm{s}} \\
\mathrm{K} \\
\mu\end{array}$ & $\begin{array}{l}1.88 \\
0.29 \\
0.62 \\
0.31\end{array}$ & $\begin{array}{l}1.82 \\
0.28 \\
0.64 \\
0.32 \\
\text { exture: }\end{array}$ & $\begin{array}{l}1.81 \\
0.28 \\
0.66 \\
0.34 \\
\text { roporph }\end{array}$ & $\begin{array}{l}1.79 \\
0.27 \\
0.67 \\
0.36 \\
\text { dolerit }\end{array}$ & $\begin{array}{l}1.79 \\
0.28 \\
0.70 \\
0.37\end{array}$ & $\begin{array}{l}\text { Unit } 240 \text { Piece } 2 \text {, slightly alt; highly alt near } \\
\text { halos to act, chl, alb, and titanite with minor } \\
\text { epidote. }\end{array}$ \\
\hline $\begin{array}{l}\text { 140-504B-209R-2 (124-126) } \\
\quad \begin{array}{l}\rho=2.92 \mathrm{~g} / \mathrm{cm}^{3} \\
\phi=1.43 \%\end{array} \\
\text { Mod OI Plag Cpx Phyric Diabase }\end{array}$ & $\begin{array}{l}\mathrm{V}_{\mathrm{p}} / \mathrm{V}_{\mathrm{s}} \\
\mathrm{K} \\
\mu\end{array}$ & $\begin{array}{l}1.88 \\
0.30 \\
0.73 \\
0.33\end{array}$ & $\begin{array}{r}1.86 \\
0.30 \\
0.74 \\
0.35 \\
\text { xture: }\end{array}$ & $\begin{array}{l}1.84 \\
0.29 \\
0.74 \\
0.36 \\
\text { roporph }\end{array}$ & $\begin{array}{l}1.81 \\
0.28 \\
0.73 \\
0.38 \\
\text { dolerit }\end{array}$ & $\begin{array}{l}1.81 \\
0.28 \\
0.74 \\
0.38\end{array}$ & $\begin{array}{l}\text { Unit } 240 \text { Piece } 20 \text {, slightly alt; highly alt near } \\
\text { halos to act, chl, alb, and titanite with minor } \\
\text { epidote. }\end{array}$ \\
\hline 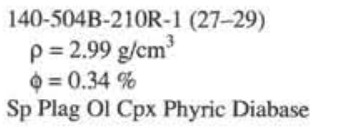 & $\begin{array}{l}\mathrm{V}_{\mathrm{p}} \mathrm{V}_{\mathrm{s}} \\
\mathrm{K} \\
\mu\end{array}$ & $\begin{array}{r}1.95 \\
0.32 \\
0.73 \\
0.31 \\
T\end{array}$ & $\begin{array}{r}1.90 \\
0.30 \\
0.75 \\
0.34 \\
\text { Ire: fine }\end{array}$ & $\begin{array}{c}1.86 \\
0.30 \\
0.77 \\
0.36 \\
\text { aed equi }\end{array}$ & $\begin{array}{c}1.83 \\
0.29 \\
0.79 \\
0.39 \\
\text { dar dol }\end{array}$ & $\begin{array}{l}1.82 \\
0.29 \\
0.81 \\
0.40\end{array}$ & $\begin{array}{l}\text { Unit } 241 \text { Piece } 4 \mathrm{~B} \text {, slightly alt to act, chl, alb. } \\
\text { and titanite. Ol alt to act, mcl, and pyrite. }\end{array}$ \\
\hline
\end{tabular}


Table 4 (continued).

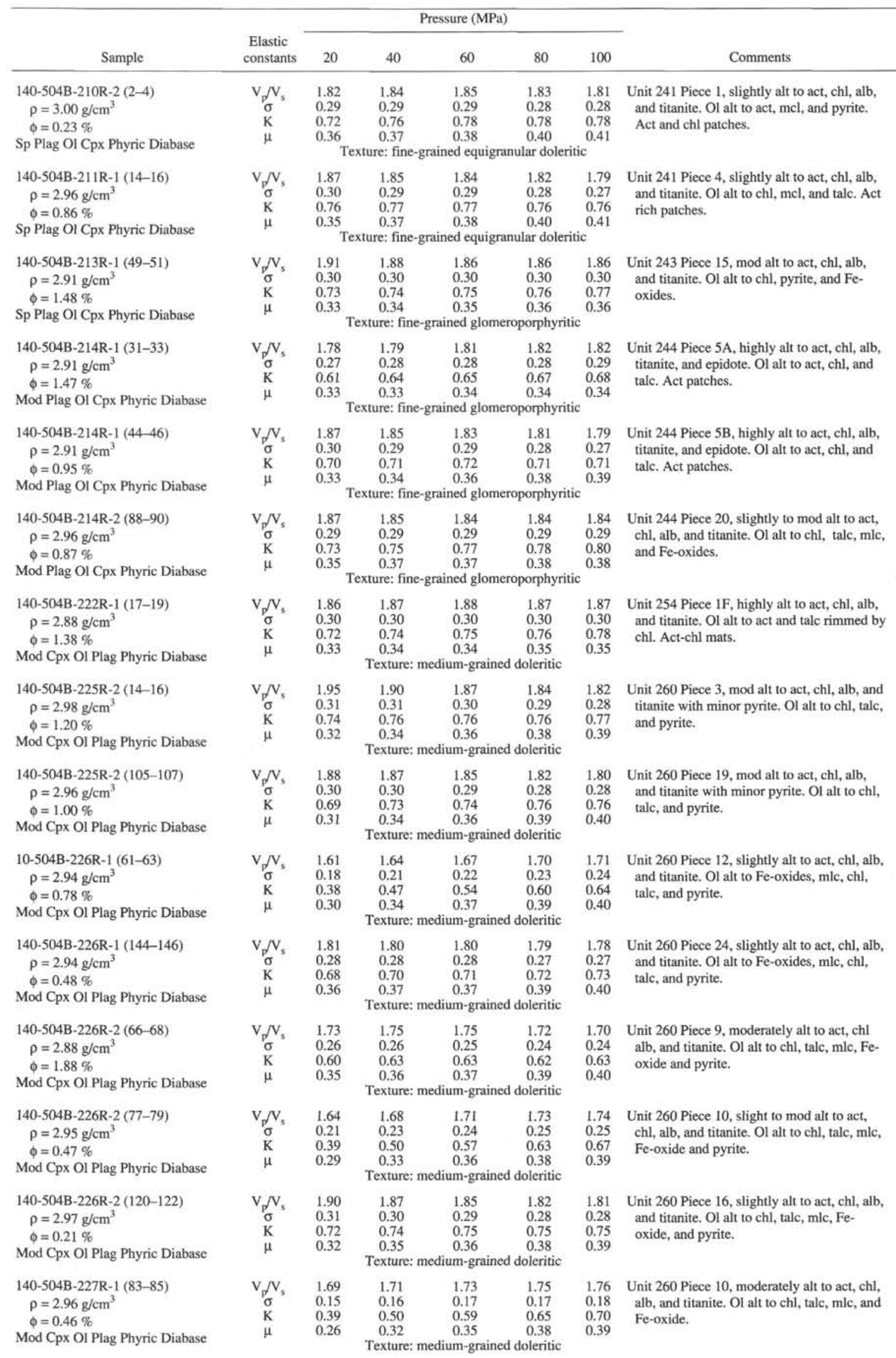


Table 4 (continued).

\begin{tabular}{|c|c|c|c|c|c|c|c|}
\hline \multirow[b]{2}{*}{ Sample } & \multirow[b]{2}{*}{$\begin{array}{l}\text { Elastic } \\
\text { constants }\end{array}$} & \multicolumn{5}{|c|}{ Pressure (MPa) } & \multirow[b]{2}{*}{ Comments } \\
\hline & & 20 & 40 & 60 & 80 & 100 & \\
\hline $\begin{array}{l}\text { 140-504B-236R-1 (32-34) } \\
\qquad \begin{array}{l}\rho=2.87 \mathrm{~g} / \mathrm{cm}^{3} \\
\phi=2.96 \%\end{array} \\
\text { Mod Plag Ol Cpx Phyric Diabase }\end{array}$ & $\begin{array}{l}\mathrm{V}_{\mathrm{p}} / \mathrm{V}_{\mathrm{s}} \\
\mathrm{K} \\
\mu\end{array}$ & $\begin{array}{l}1.80 \\
0.26 \\
0.63 \\
0.36\end{array}$ & $\begin{array}{l}1.76 \\
0.26 \\
0.65 \\
0.37 \\
\text { Textu }\end{array}$ & $\begin{array}{c}1.76 \\
0.27 \\
0.67 \\
0.37 \\
\text { e-grain }\end{array}$ & $\begin{array}{r}1.77 \\
0.27 \\
0.69 \\
0.37 \\
\text { eritic }\end{array}$ & $\begin{array}{l}1.79 \\
0.27 \\
0.71 \\
0.38\end{array}$ & $\begin{array}{l}\text { Unit } 269 \text { Piece } 5 \mathrm{~B} \text {, highly alt to act, chl, alb, } \\
\text { and titanite. Ol alt to chl, pyrite, and Fe- } \\
\text { oxides. Fine act amygs. }\end{array}$ \\
\hline $\begin{array}{l}\text { 140-504B-236R-1 (53-55) } \\
\qquad \begin{array}{l}\rho=2.93 \mathrm{~g} / \mathrm{cm}^{3} \\
\phi=0.40 \%\end{array} \\
\text { Mod Plag Ol Cpx Phyric Diabase }\end{array}$ & $\begin{array}{l}\mathrm{V}_{\mathrm{p}} / \mathrm{V}_{\mathrm{s}} \\
\sigma \\
\mathrm{K} \\
\mu\end{array}$ & $\begin{array}{l}1.91 \\
0.31 \\
0.71 \\
0.32\end{array}$ & $\begin{array}{l}1.88 \\
0.30 \\
0.73 \\
0.34 \\
\text { Textu }\end{array}$ & $\begin{array}{r}1.86 \\
0.29 \\
0.74 \\
0.35 \\
\text { e-grain }\end{array}$ & $\begin{array}{r}1.83 \\
0.28 \\
0.74 \\
0.38 \\
\text { eritic }\end{array}$ & $\begin{array}{l}1.81 \\
0.28 \\
0.75 \\
0.39\end{array}$ & $\begin{array}{l}\text { Unit } 269 \text { Piece } 5 \mathrm{~B} \text {, highly alt to act, chl, alb, } \\
\text { and titanite. Ol alt to chl, pyrite, and Fe- } \\
\text { oxides. Fractures. }\end{array}$ \\
\hline
\end{tabular}

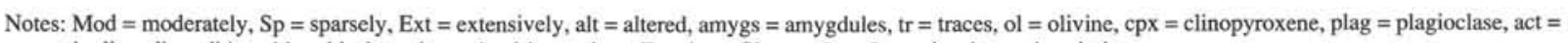
actinolite, $\mathrm{alb}=$ albite, $\mathrm{chl}=$ chlorite, $\mathrm{mlc}=$ mixed-layer clays, $\mathrm{Fe}=$ iron. Observations from visual core descriptions.

tions seem to be compositionally and structurally similar to the rocks from Hole 504B.

The northern section of the Oman ophiolite is characterized by hydrous andesitic magmatism and high- pressure metamorphism with an upper crustal section composed of upper and lower pillow lavas (Christensen and Smewing; 1981; Nicolas, 1989). The origin of the Oman ophiolite has been the subject of a dispute between supporters of island arc or back-arc basin environments (Pearce et al., 1981; Alabaster et. al., 1982; Searle and Stevens; 1984; Lippard et al., 1986; Beurrier, 1988; Le Métour, 1987) and other workers who support a model involving accretion at a mid-ocean ridge (Boudier et al., 1985, 1988; Ernewein, 1988; Nicolas, 1989). Nevertheless, both groups have documented the existence of secondary magmatism within this ophiolitic section. Therefore, physical properties from the Oman ophiolite complex and Hole 504B were compared to relate midocean-ridge upper crustal rocks with a system that seems to be influenced by offridge volcanism.

A comparison of $V_{p}$ and $V_{s}$ as a function of depth with ophiolite data from the Bay of Islands North Arm (Christensen and Salisbury, 1982) and Blow Me Down (Salisbury and Christensen, 1978) massifs as well as the northern section of the Oman complex (Christensen and Smewing, 1981) display similar trends of increasing velocity with depth (Fig. 12). However, statistical analyses of the data show that the mean of in-situ $V_{p}$ and $V_{s}$ for Layer $2 \mathrm{~B}$ agree with the results from the Blow Me down massif (Figs. 12A and B) but are somewhat higher than the results from the North Arm and the Oman ophiolites (Figs. $12 \mathrm{C}$ and $12 \mathrm{D}$ ). In the sheeted dikes section, the average in-situ $V_{p}$ of Hole 504B is lower than those from the Bay of Islands massifs and higher than the value from the Oman ophiolite, whereas the in-situ $V_{s}$ mean value is similar to the values obtained from the Bay of Islands samples and significantly higher than the mean of the Oman section (Fig. 12). The variations in density with depth for the same regions show that Hole 504B has a higher mean bulk-density value for both layers $2 \mathrm{~B}$ and $2 \mathrm{C}$ than any of the previously published ophiolite data (Fig. 13). The number of density measurements in Hole 504B (Fig. $13 \mathrm{~A}$ ) is significantly greater than the samples used in any of the previous studies; hence, the differences might be due to having a more detailed sampling coverage in Hole 504B. Nevertheless, the values obtained from the Bay of Islands samples (Figs. 13B and 13C) are within the range displayed by the Hole 504B samples, whereas the study in the Oman ophiolite has significantly lower values (Fig. 13D).

Overall, physical properties observations indicate that the samples from Hole 504B tend to correlate favorably with the samples from the Bay of Islands but not with the Oman complex. The minor discrepancies in density with the Bay of Islands can be attributed to weathering effects of the exposed ophiolitic suites, whereas the more significant differences in velocities and densities with the Oman section are thought to be related to the different geochemical compositions and tectonic histories between these areas. Similar physical properties studies of the Oman ophiolite rocks also suggested that the signifi- cantly lower velocities and densities are because of different geochemical compositions (Christensen et al., 1989).

\section{SUMMARY}

Measurements of porosity, density, $V_{p}$, and $V_{s}$ from samples collected from Hole 504B, Legs 137 and 140, reveal the following conclusions regarding the seismic properties of deep oceanic crustal rocks and the nature of the base of the sheeted dike complex:

1. Crack porosity within the rocks of Hole 504B tends to reduce seismic velocities at low pressures. The constant increase of in-situ pressure velocity measurements with depth differs significantly from the atmospheric pressure measurements performed during the last several ODP legs. The release of in-situ stresses due to unloading as the samples are brought to the surface from deep crustal sections causes large amounts of fracturing in the rocks, hence affecting the physical properties of the samples.

2. Large fracture zones are thought to be present at approximately $1650 \mathrm{mbsf}$ and between $1800 \mathrm{mbsf}$ and $1850 \mathrm{mbsf}$. These zones may have contributed or may still contribute as pathways of hydrothermal circulation. The shallow laterolog information and the mineralogy from these sections tend to support these findings.

3. Low velocity zones correlating with the fracture zones may be responsible for intracrustal reflections. These zones are controlled by hydrothermal systems that cause rock alteration, lowering the bulk density and perhaps providing the necessary acoustic impedance contrast to produce significant reflectors.

4. The samples from Hole 504B fall in a region bounded by lines of constant mean atomic weight between $\mathrm{m}=21$ and $\mathrm{m}=22$ with a few exceptions. The samples that fall outside of this area belong to the zones characterized by a transition between oxic and anoxic types of alteration and tend to be different from the samples found in the deeper sections of the hole.

5. $V_{p} / V_{s}$ and Poisson's ratios decrease, whereas shear and bulk moduli generally increase as a function of depth. Crack aspect ratios, crack density, and a decrease in porosity with depth recorded in logging data and laboratory samples provide explanations for the variations in elastic constants.

6. Physical properties data from the Bay of Islands ophiolite agree well with data from Hole $504 \mathrm{~B}$, whereas physical properties data from the Oman ophiolite contrast significantly with data from Hole 504B. Some of the minor differences are attributed to sampling density, whereas the larger discrepancies observed in the Oman rocks might be related to different tectonic histories and the production of more andesitic compositions by offridge volcanism.

\section{ACKNOWLEDGMENTS}

This research was funded partially by the Joint Oceanographic Institutions, United States Science Advisory Committee (JOI/USSAC) 


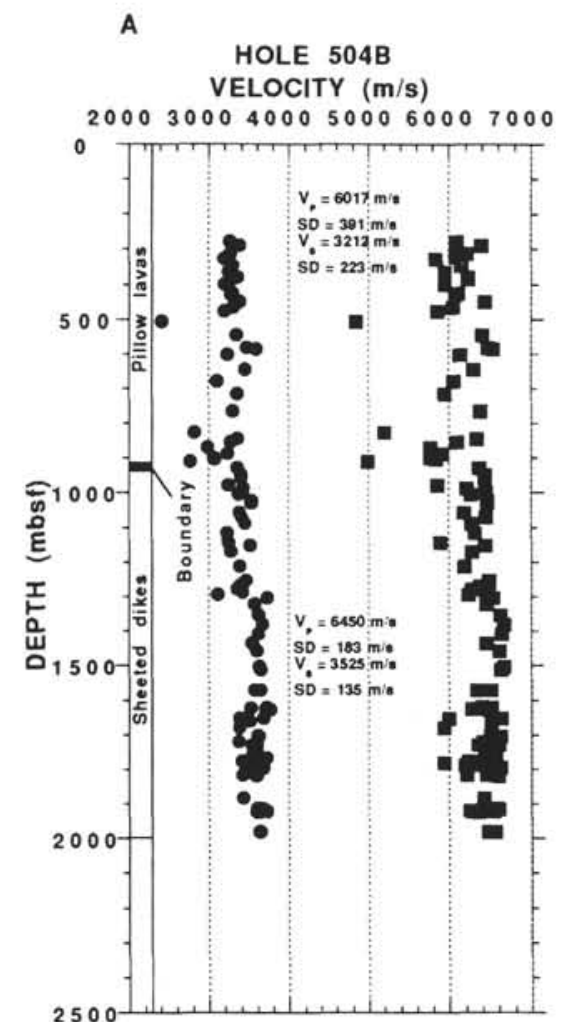

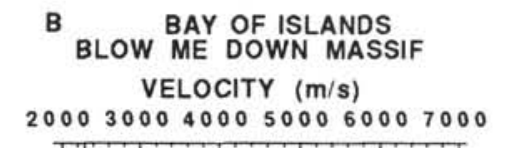

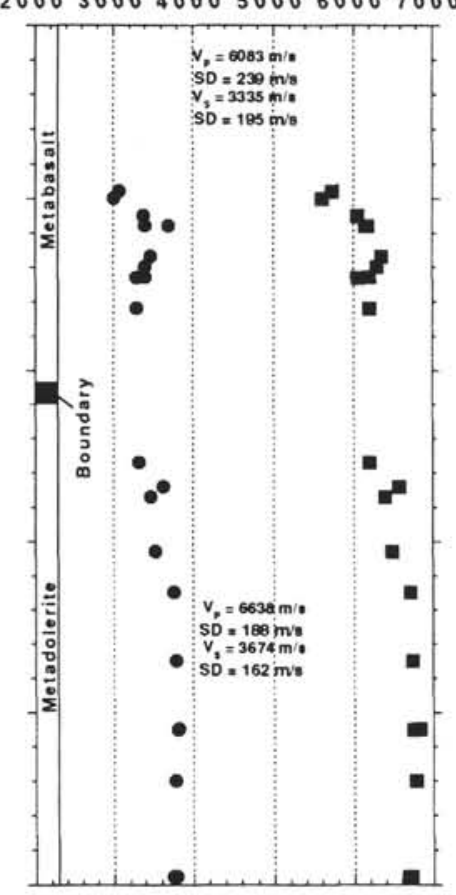

C NORTH OF ISLANDS $\operatorname{VELOCITY}(\mathrm{m} / \mathrm{s})$

200030004000500060007000

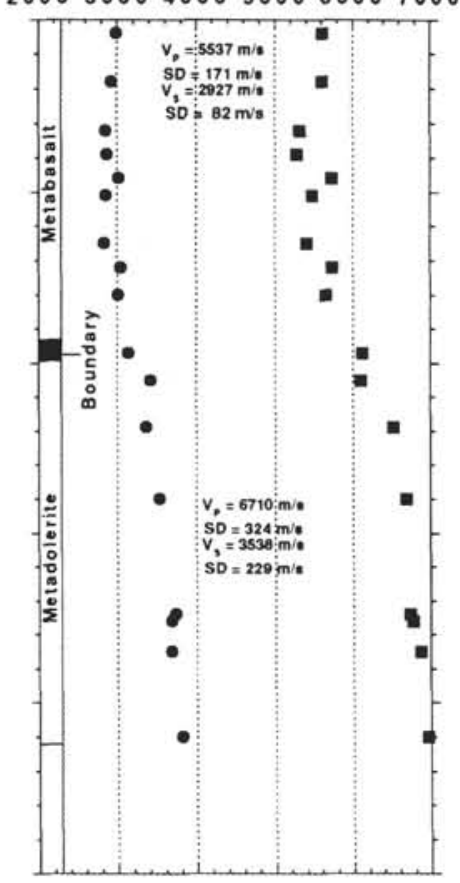
D OMAN OPHIOLITE

VELOCITY $(\mathrm{m} / \mathrm{s})$

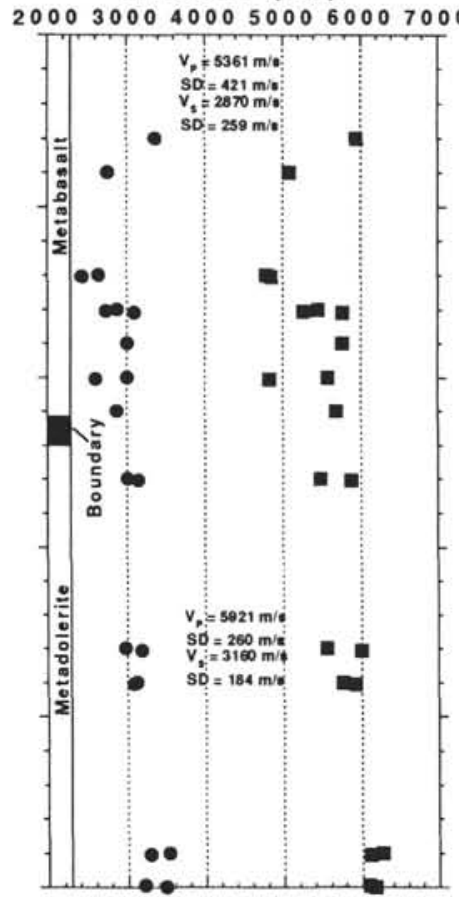

Figure 12. A plot of in-situ $V_{\nu}$ (squares) and $V_{s}$ (circles) as a function of depth for samples from (A) Hole 504B; (B) Bay of Islands Blow Me Down massif; (C) Bay of Islands North Arm massif; (D) northern section of the Oman ophiolite. SD denotes standard deviation, whereas $V_{p}$ and $V_{s}$ represent the average compressional-and shear-wave velocities, respectively, for the particular section. 


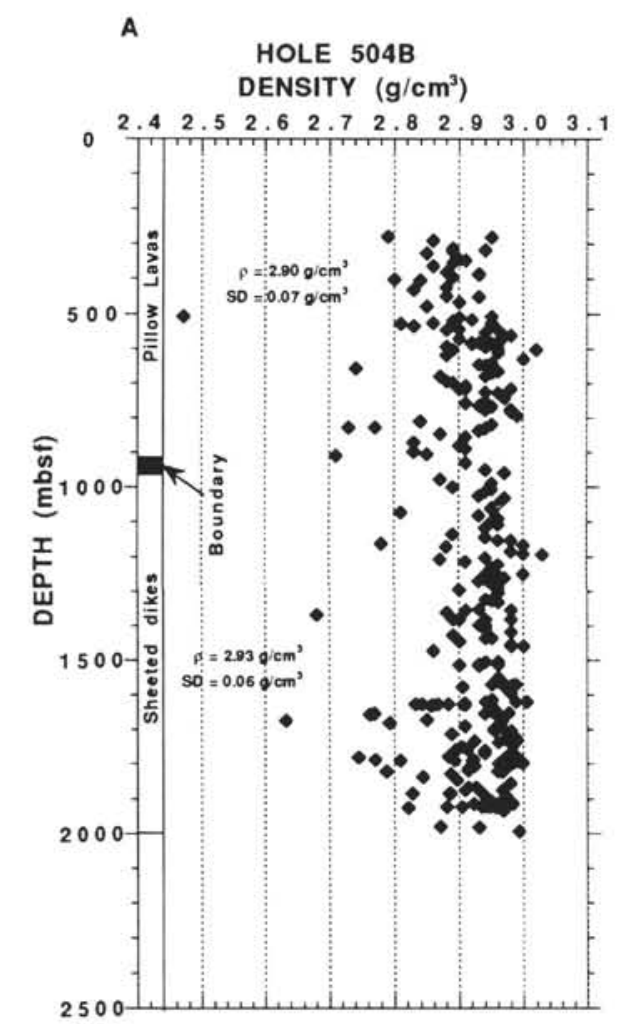

B BYY OF ISLANDS
BLOW ME DOWN MASSIF DENSITY $(\mathrm{g} / \mathrm{cm} 3)$

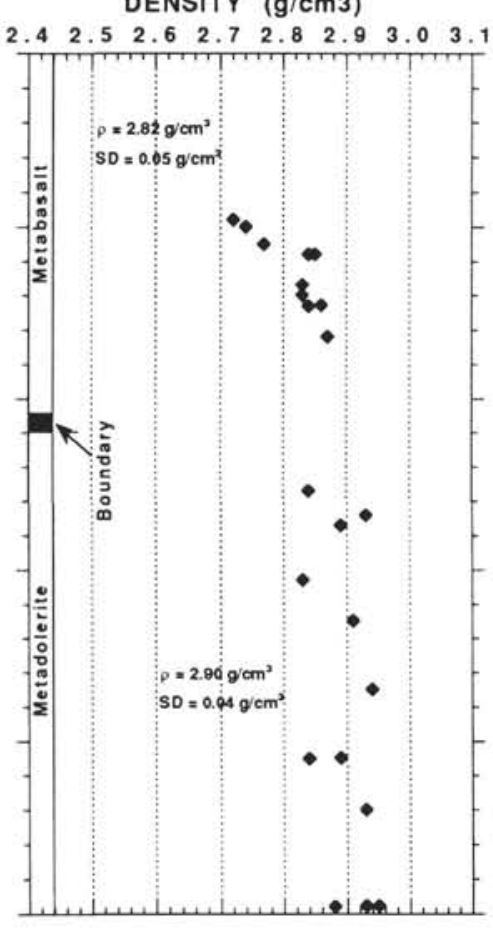

c NORTH ARM MASSIF

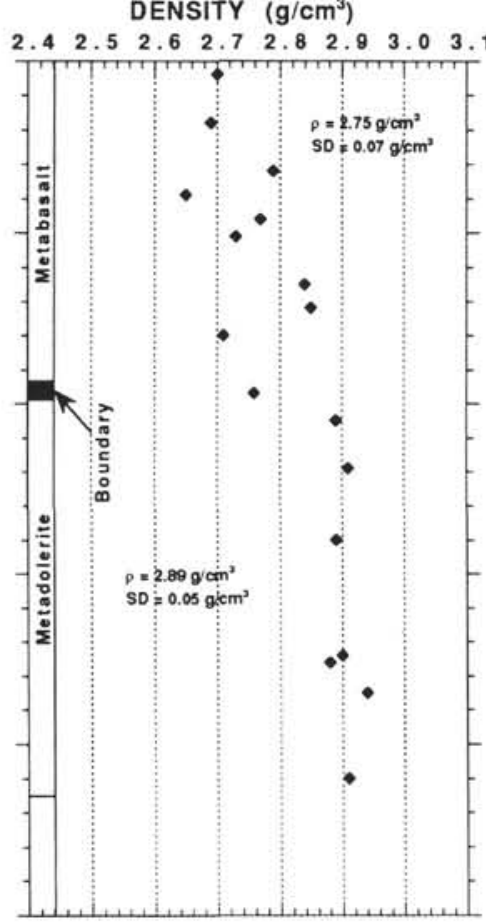

D OMAN OPHIOLITE NORTHERN SECTION DENSITY $\left(\mathrm{g} / \mathrm{cm}^{3}\right)$

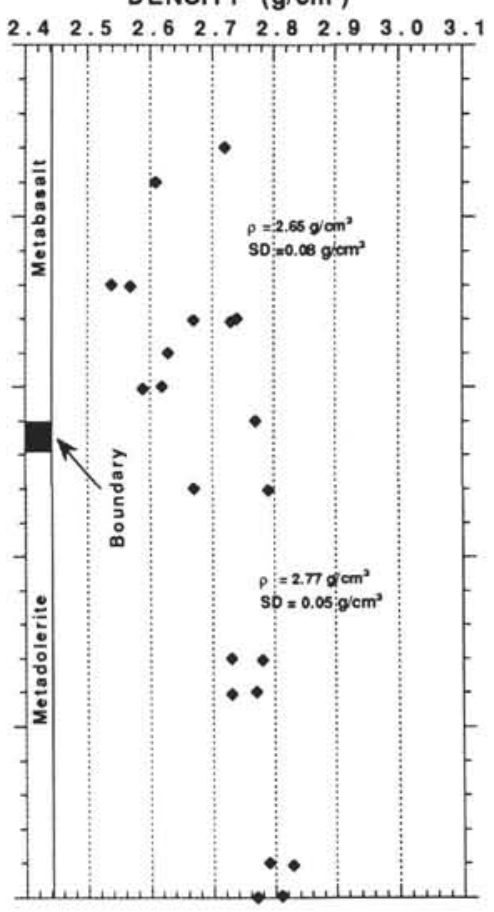

Figure 13. Density as a function of depth for samples from (A) Hole 504B; (B) Bay of Islands Blow Me Down massif; (C) Bay of Islands North Arm massif; (D) northern section of the Oman ophiolite. SD denotes standard deviation, whereas $\rho$ represents the average density for that section. 
and the Office of Naval Research (ONR). We wish to thank Dr. Robert L. Nowack, Dr. Peter M. Shearer, Dr. Christopher Harrison, and an anonymous reviewer for their helpful criticisms of this manuscript. Special thanks to the Geological Survey of Denmark and Dr. Janet Pariso for helping with porosity and density measurements as well as to Dr. Gregor Eberli for permitting the use of the RSMAS high pressure-velocity system. We also thank the captain, operations superintendent, core technicians, and drill crew of the JOIDES Resolution.

\section{REFERENCES}

Alabaster, T., Pearce, J.A., and Malpas, J., 1982. The volcanic stratigraphy and petrogenesis of the Oman ophiolite complex. Contrib. Mineral. Petrol., $81: 168-13$.

Alt, J.C., and Emmermann, R., 1985. Geochemistry of hydrothermally altered basalts: Deep Sea Drilling Project Hole 504B, Leg 83. In Anderson, R.N., Honnorez, J., Becker, K., et al., Init. Repts. DSDP, 83: Washington (U.S. Govt. Printing Office), 249-262.

Alt, J.C., Honnorez, J., Laverne, C., and Emmermann, R., 1986. Hydrothermal alteration of a $1 \mathrm{~km}$ section through the upper oceanic crust, Deep Sea Drilling Project Hole 504B: mineralogy, chemistry, and evolution of seawater-basalt interactions. J. Geophys. Res., 91:10309-10335.

Alt, J.C., Laverne, C., and Muehlenbachs, K., 1985. Alteration of the upper oceanic crust: mineralogy and processes in Deep Sea Drilling Project Hole 504B, Leg 83. In Anderson, R.N., Honnorez, J., Becker, K., et al., Init. Repts. DSDP, 83: Washington (U.S. Govt. Printing Office), 217-247.

Anderson, R.N., and Zoback, M.D., 1982. Permeability, underpressures, and convection in the oceanic crust near the Costa Rica Rift, eastern equatorial Pacific. J. Geophys. Res., 87:2860-2868.

Anderson, R.N., Zoback, M.D., Hickman, S.H., and Newmark, R.L., 1985. Permeability versus depth in the upper oceanic crust: in situ measurements in Deep Sea Drilling Project Hole 504B, eastern equatorial Pacific. In Anderson, R.N., Honnorez, J., et al., Init. Repts. DSDP, 83: Washington (U.S. Govt. Printing Office), 429-442.

ASTM, 1989. Annual Book of ASTM Standards for Soil and Rock; Building Stones: Geotextiles (Vol. 04.08): Philadelphia (Am. Soc. Testing Materials).

Au, D., and Clowes, R.M., 1984. Shear-wave velocity structure of the oceanic lithosphere from ocean bottom seismometer studies. Geophys. J. R. Astron. Soc., 77:105-123.

Baragar, W.R.A., Lambert, M.B., Baglow, N., and Gibson, I.L., 1989. Sheeted dikes from CY-4 and surface sections: Troodos Ophiolite. In Gibson, I.L., Malpas, J., Robinson, P.T., and Xenophontos, C. (Eds.), Cyprus Crustal Study Project: Initial Report, Hole CY-4. Geol. Surv. Can., 69-106.

Becker, K., 1985. Large-scale electrical resistivity and bulk porosity of the oceanic crust, Deep Sea Drilling Project Hole 504B, Costa Rica Rift. In Anderson, R.N., Honnorez, J., Becker, K., et al., Init. Repts. DSDP, 83: Washington (U.S. Govt. Printing Office), 419-427.

, 1989. Measurements of the permeability of the sheeted dikes in Hole 504B, ODP Leg 111. In Becker, K., Sakai, H., et al., Proc. ODP, Sci. Results, 111: College Station, TX (Ocean Drilling Program), 317-325.

Becker, K., Foss, G., et al., 1992. Proc. ODP, Init. Repts., 137: College Station, TX (Ocean Drilling Program).

Becker, K., Langseth, M.G., and Von Herzen, R.P., 1983. Deep crustal geothermal measurements. Hole 504B. Deep Sea Drilling Project Legs 69 and 70. In Cann, J.R., Langseth, M.G., Honnorez, J., Von Herzen, R.P., White, S.M., et al., Init. Repts. DSDP, 69: Washington (U.S. Govt. Printing Office), 223-236.

Becker, K., Langseth, M.G., Von Herzen, R.P., and Anderson, R.N., 1983. Deep crustal geothermal measurements, Hole 504B, Costa Rica Rift. J. Geophys. Res., 88:3447-3457.

Becker, K., Langseth, M.G., Von Herzen, R.P., Anderson, R.N., and Hobart, M.A., 1985. Deep crustal geothermal measurements, Hole 504B, Deep Sea Drilling Project Legs 69, 70, 83, and 92. In Anderson, R.N., Honnorez, J., Becker, K., et al., Init. Repts. DSDP, 83: Washington (U.S. Govt. Printing Office), 405-418.

\footnotetext{
-Abbreviations for names of organizations and publications in ODP reference lists follow the style given in Chemical Abstracts Service Source Index (published by American Chemical Society).
}

Becker, K., Sakai, H., et al., 1988. Proc. ODP, Init. Repts., 111: College Station, TX (Ocean Drilling Program).

Beurrier, M., 1988. Géologie de la nappe ophiolitique de Samail dans les parties orientale et centrale des montagnes d'Oman [thèse doc. etat]. Univ. Paris VI, Paris.

Birch, F., 1960. The velocity of compressional waves in rocks to 10 kilobars, 1. J. Geophys. Res., 65:1083-1102.

, 1961. The velocity of compressional waves in rocks to 10 kilobars, 2. J. Geophys. Res., 66:2199-2224.

Boudier, F., Bouchez, J.L., Nicolas, A., Cannat, M., Ceuleneer, G., Misseri, M., and Montigny, R., 1985. Kinematics of oceanic thrusting in the Oman ophiolite: model of plate convergence. Earth Planet. Sci. Lett., 76:84-92.

Boudier, F., Ceuleneer, G., and Nicolas, A., 1988. Shear zones, thrusts and related magmatism in the Oman ophiolite: initiation of thrusting on an oceanic ridge. Tectonophysics, 151:275-296.

Cann, J.R., Langseth, M.G., Honnorez, J., Von Herzen, R.P., White, S.M., et al., 1983. Init. Repts. DSDP, 69: Washington (U.S. Govt. Printing Office).

Casey, J.F., Elthon, D.L., Sirkoy, F.X., Karson, J.A., and Sullivan, J., 1985. Geochemical and geological evidence bearing on the origin of the Bay of Islands and Coastal Complex ophiolites of western Newfoundland. Tectonophysics, 116:1-40.

Christensen, N.I., 1965. Compressional-wave velocities in metamorphic rocks at pressures to 10 kilobars. J. Geophys. Res., 70:6147-6164.

- , 1979. Compressional-wave velocities in rocks at high temperatures and pressures, critical thermal gradients, and crustal low velocity zones. $J$. Geophys. Res., 84:6849-6858.

, 1984. Pore pressure and oceanic crustal seismic structure. Geophys. J. R. Astron. Soc., 79:411-424.

1985. Measurements of dynamic properties of rocks at elevated temperatures and pressures. In Pincus, H.J., and Hoskins, E.R. (Eds.), Measurements of Rock Properties at Elevated Pressures and Temperatures: Philadelphia (Am. Soc. for Testing and Materials), ASTM STP 869:93-107.

Christensen, N.I., and Salisbury, M.H., 1982. Lateral heterogeneity in the seismic structure of the ocean crust inferred from velocity studies in the Bay of Islands ophiolite, Newfoundland. Geophys. J. R. Astron. Soc., 68:675-688.

, 1985. Seismic velocities, densities and porosities of Layer $2 \mathrm{~B}$ and Layer 2C basalts from Hole 504B. In Anderson, R.N., Honnorez, J., Becker, K., et al., Init. Repts. DSDP, 83: Washington (U.S. Govt. Printing Office), 367-370.

1989. Velocity structure of the Troodos Massif, an arc-derived ophiolite. In Gibson, I.L., Malpas, J., Robinson, P.T., and Xenophontos, C. (Eds.), Cyprus Crustal Study Project: Initial Report, Hole CY-4. Geol. Surv. Can., 351-369.

Christensen, N.I., and Smewing, J., 1981. Geology and seismic structure of the northern section of the Oman ophiolite. J. Geophys. Res., 86:25452555.

Christensen, N.I., Wepfer, W.W., and Baud, R.D., 1989. Seismic properties of sheeted dikes from Hole 504B, ODP Leg 111. In Becker, K., Sakai, H., et al., Proc. ODP, Sci. Results, 111: College Station, TX (Ocean Drilling Program), 171-176.

Collins, J.A., Brocher, T.M., and Purdy, G.M., 1989. Seismic-reflection structure of the upper oceanic crust: implications from DSDP/ODP Hole 504B, Panama Basin. In Becker, K., Sakai, H., et al., Proc. ODP, Sci. Results, 111: College Station, TX (Ocean Drilling Program), 177-191.

Dick, H.J.B., Erzinger, J., Stokking, L.B., et al., 1992. Proc. ODP, Init. Repts., 140: College Station, TX (Ocean Drilling Program).

Elthon, D., Casey, J.F., and Komor, S., 1982. Mineral chemistry of ultramafic cumulates from the North Arm massif of the Bay of Islands ophiolite: implication for high pressure fractionation of oceanic basalts. J. Geophys. Res., 87:8717-8734.

, 1984. Cryptic mineral chemistry variations in a detailed traverse through the cumulate ultramafic rocks of the North Arm massif of the Bay of Islands ophiolite complex. Geol. Soc. Spec. Publ. London, 13:185-197.

Emmermann, R., 1985. Basement geochemistry, Hole 504B. In Anderson, R.N., Honnorez, J., Becker, K., et al., Init. Repts. DSDP, 83: Washington (U.S. Govt. Printing Office), 183-199.

Ernewein, M., Pflumio, C., and Whitechurch, H., 1988. The death of an accretion zone as evidenced by the magmatic history of the Sumail Ophiolite (Oman). Tectonophysics, 151:247-274.

Fisher, A.T., Becker, K., Narasimhan, T.N., Langseth, M.G., and Mottl, M.J., 1990. Passive, off-axis convection through the southern flank of the Costa Rica rift. J. Geophys. Res., 95:9343-9370. 
Gable, R., Morin, R.H., and Becker, K., 1989. Geothermal state of Hole 504B: ODP Leg 111 overview. In Becker, K., Sakai, H., et al., Proc. ODP, Sci. Results, 111: College Station, TX (Ocean Drilling Program), 87-96.

Garbin, H.D., and Knopoff, L., 1975. Elastic moduli of a medium with liquid-filled cracks. Q. Appl. Math., 33:301-303.

Hobart, M.A., Langseth, M.G., and Anderson, R.N., 1985. A geothermal and geophysical survey on the south flank of the Costa Rica Rift: Sites 504 and 505. In Anderson, R.N., Honnorez, J., et al., Init. Repts. DSDP, 83: Washington (U.S. Govt. Printing Office), 379-404.

Hudson, J.A., 1980. Wave speeds and attenuation of elastic waves in material containing cracks. Geophys. J. R. Astron. Soc., 64:133-150.

Hyndman, R.D., 1979. Poisson's ratio in the oceanic crust-a review. Tectonophysics, 59:321-333.

Hyndman, R.D., and Drury, M.J., 1976. The physical properties of oceanic basement rocks from deep drilling on the Mid-Atlantic Ridge at $23^{\circ} \mathrm{N} . J$. Geophys. Res., 81:4042-4052.

Iturrino, G.J., and Christensen, N.I., 1991. Reflectivity modeling of the layer 3 gabbroic sequence drilled at the Atlantis II Fracture Zone. In Von Herzen, R.P., Robinson, P.T., et al., Proc. ODP, Sci. Results, 118: College Station, TX (Ocean Drilling Program), 245-252.

Iturrino, G.J., Christensen, N.I., Kirby, S., and Salisbury, M.H., 1991. Seismic velocities and elastic properties of oceanic gabbroic rocks from Hole 735B. In Von Herzen, R.P., Robinson, P.T., et al., Proc. ODP, Sci. Results, 118: College Station, TX (Ocean Drilling Program), 227-244.

Jacobsen, S.B., and Wasserburg, G.J., 1979. Nd and Sr isotopic study of the Bay of Islands ophiolite complex and the evolution of the source of mid-ocean ridge basalts. J. Geophys. Res., 84:7429-7445.

Karato, S., 1983. Physical properties of basalts from Deep Sea Drilling Project Hole 504B, Costa Rica Rift. In Cann, J.R., Langseth, M.G., Honnorez, J., Von Herzen, R.P., White, S.M., et al., Init. Repts. DSDP, 69: Washington (U.S. Govt. Printing Office), 687-695.

Karato, S., Wilkens, R.H., and Langseth, M.G., 1983. Shipboard physicalproperties measurements of basalts from the Costa Rica Rift, Deep Sea Drilling Project Legs 69 and 70. In Cann, J.R., Langseth, M.G., Honnorez, J., Von Herzen, R.P., White, S.M., et al., Init. Repts. DSDP, 69: Washington (U.S. Govt. Printing Office), 675-681.

Kempner, W.C., and Gettrust, J.F., 1982. Ophiolites, synthetic seismograms, and ocean crustal structure: comparison of ocean bottom seismometer data and synthetic seismograms for the Bay of Islands Ophiolite. J. Geophys. Res., 87:8447-8462.

Kuster, G.T., and Toksöz, M.N., 1974. Velocity and attenuation of seismic waves in two-phase media: Part I-theoretical formulations. Geophysics, 39:587-606.

Langseth, M.G., Cann, J.R., Natland, J.H., and Hobart, M., 1983. Geothermal phenomena at the Costa Rica Rift: background and objectives for drilling at Deep Sea Drilling Project Sites 501, 504, and 505. In Cann, J.R. Langseth, M.G., Honnorez, J., Von Herzen, R.P., White, S.M., et al., Init. Repts. DSDP, 69: Washington (U.S. Govt. Printing Office), 5-29.

Langseth, M.G., Mottl, M.J., Hobart, M.A., and Fisher, A., 1988. The distribution of geothermal and geochemical gradients near Site 501/504: implications for hydrothermal circulation in the oceanic crust. In Becker. K., Sakai, H., et al., Proc. ODP, Init. Repts., 111: College Station, TX (Ocean Drilling Program), 23-32.

LeMétour, J., 1987. Géologie de l'autochtone des montagnes d’Oman dans la fenêtre du Saih Hatat [thèse doc. etat]. Univ. Paris, Paris.

Lippard, S.J., Shelton, A.W., and Gass, I.G., 1986. The Ophiolite of Northern Oman. Geol. Soc. London Mem., 11.
Malpas, J., 1977. Petrology and tectonic significance of Newfoundland ophiolites with examples from the Bay of Islands. Bull.-Oreg., Dep. Geol. Miner. Ind., 95: 13-23.

1978. Magma generation in the upper mantle, field evidence from ophiolite suites and application to the generation of oceanic lithosphere. Philos. Trans. R. Soc. London A, 288:527-546.

Nicolas, A., 1989. Structure of Ophiolites and Dynamics of the Oceanic Lithosphere: Dordrecht (Kluwer).

O'Connell, R.J., and Budianski, B., 1974. Seismic velocities in dry and saturated cracked solids. J. Geophys. Res., 79:5412-5426.

Parsons, B., and Sclater, J.G., 1977. An analysis of the variation of ocean floor bathymetry and heat flow with age. J. Geophys. Res., 82:803-829.

Pearce, J.A.. Alabaster, T., Shelton, A.W., and Searle, M.P., 1981. The Oman ophiolite as a Cretaceous arc-basin complex: evidence and implications. Philos. Trans. R. Soc. London A, 300:299-317.

Pezard, P.A., and Anderson, R.N., 1989. Morphology and alteration of the upper oceanic crust from in-situ electrical experiments in DSDP/ODP Hole 504B. In Becker, K., Sakai, H., et al., Proc. ODP, Sci. Results, 111: College Station, TX (Ocean Drilling Program), 133-146.

Robinson, P.T., 1989. Cyprus Crustal Study Project Hole CY-4: lithologic unit summaries. In Gibson, I.L., Malpas, J., Robinson, P.T., and Xenophontos, C. (Eds.), Cyprus Crustal Study Project: Initial Report, Hole CY-4. Geol. Surv. Can., 5-29.

Robinson, P.T., Gibson, I.L., and Horne, L.V.B., 1991. Cyprus Crustal Study Project Hole CY-1: lithologic unit summaries. In Gibson, I.L., Malpas, J., Robinson, P.T., and Xenophontos, C. (Eds.), Cyprus Crustal Study Project: Initial Report, Hole CY-4. Geol. Surv. Can., 5-28.

Salisbury, M.H., and Christensen, N.I., 1978. The seismic velocity structure of a traverse through the Bay of Islands ophiolite complex, Newfoundland: an exposure of oceanic crust and upper mantle. J. Geophys. Res., 83:805817.

Searle, M.P., and Stevens, R.K., 1984. Obduction processes in ancient, modern and future ophiolites. Geol. Soc. Spec. Publ. London, 13:303-320.

Shearer, P.M., 1988. Cracked media, Poisson's ratio and the structure of the oceanic crust. J. Geophys. Res., 92:357-362.

Spudich, P., and Orcutt, J., 1980. Petrology and porosity of an oceanic crustal site: results from wave form modeling of seismic refraction data. $J$. Geophys. Res., 85:1409-1433.

Walsh, J.B., 1969. New analysis of attenuation in partially melted rock. $J$. Geophys. Res., 74:4333-4337.

Watt, J.P., Davies, G.F., and O'Connell, R.J., 1976. The elastic properties of composite materials. Rev. Geophys. Space Phys., 14:541-563.

Wilkens, R.H., Christensen, N.I., and Slater, L., 1983. High-pressure seismic studies of Leg 69 and 70 basalts. In Cann, J.R., Langseth, M.G., Honnorez, J., Von Herzen, R.P., White, S.M., et al., Init. Repts. DSDP, 69: Washington U.S. Govt. Printing Office), 683-686.

Williams, H., and Malpas, J., 1972. Sheeted dikes and brecciated dike rocks within transported igneous complexes, Bay of Islands, western Newfoundland. Can. J. Earth Sci., 9:1216-1229.

Date of initial receipt: 5 April 1993

Date of acceptance: 20 December 1993

Ms 137/140SR-031 